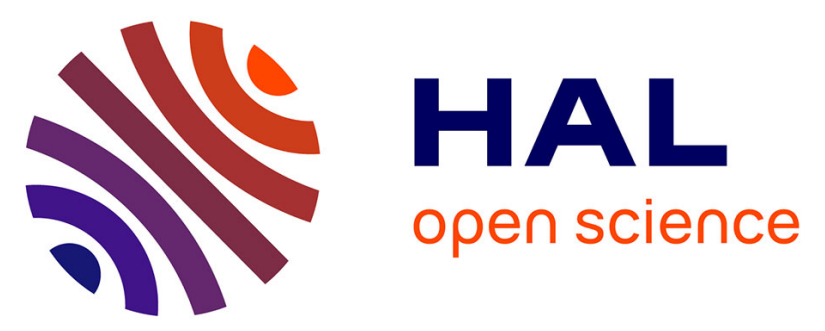

\title{
Sensitization of tumor cells to chemotherapy by natural products: a systematic review of preclinical data and molecular mechanisms
}

Raimundo Gonçalves de Oliveira Júnior, Christiane Adrielly Alves Ferraz, Jackson Roberto Guedes da Silva Almeida, Raphäel Grougnet, Valérie Thiéry, Laurent Picot

\section{To cite this version:}

Raimundo Gonçalves de Oliveira Júnior, Christiane Adrielly Alves Ferraz, Jackson Roberto Guedes da Silva Almeida, Raphäel Grougnet, Valérie Thiéry, et al.. Sensitization of tumor cells to chemotherapy by natural products: a systematic review of preclinical data and molecular mechanisms. Fitoterapia, 2018, 129, pp.383-400. 10.1016/j.fitote.2018.02.025 . hal-01807405

\section{HAL Id: hal-01807405 \\ https: / hal-univ-rochelle.archives-ouvertes.fr/hal-01807405}

Submitted on 4 Jun 2018

HAL is a multi-disciplinary open access archive for the deposit and dissemination of scientific research documents, whether they are published or not. The documents may come from teaching and research institutions in France or abroad, or from public or private research centers.
L'archive ouverte pluridisciplinaire HAL, est destinée au dépôt et à la diffusion de documents scientifiques de niveau recherche, publiés ou non, émanant des établissements d'enseignement et de recherche français ou étrangers, des laboratoires publics ou privés. 


\section{Sensitization of tumor cells to chemotherapy by natural products: a systematic review of preclinical data and molecular mechanisms}

Raimundo Gonçalves de Oliveira Júnior ${ }^{1}$, Christiane Adrielly Alves Ferraz ${ }^{2}$, Jackson Roberto Guedes da Silva Almeida ${ }^{2}$, Raphaël Grougnet ${ }^{3}$, Valérie Thiéry ${ }^{1}$, Laurent Picot $^{1 *}$

${ }^{1}$ UMRi CNRS 7266 LIENSs, University of La Rochelle, 17042 La Rochelle, France.

${ }^{2}$ Center for Studies and Research of Medicinal Plants, Federal University of San Francisco Valley, 56306-000 Petrolina, Brazil.

${ }^{3}$ UMR CNRS 8638 Laboratory of Pharmacognosy, Paris Descartes University, 75006 Paris, France.

${ }^{*}$ Corresponding author at: UMRi CNRS 7266 LIENSs, Université de La Rochelle, Curie B101 Faculté des Sciences et Technologies, Avenue Michel Crépeau, 17042 La Rochelle, France. E-mail address: laurent.picot@univ-lr.fr (L. Picot). 


\section{ABSTRACT}

Purpose: Tumor cells are spontaneously or adaptively resistant to chemotherapeutic drugs, eventually leading to the selection of multiresistant cells responsible for tumor growth and metastasis. Chemosensitization of tumor cells to conventional drugs using non-toxic natural products is a recent and innovative strategy aiming to increase the cytotoxic efficiency of anticancer drugs, limit their toxic side effects and delay the appearance of acquired chemoresistance. This systematic review summarizes data obtained from preclinical studies reporting the use of natural products to sensitize tumor cells to chemotherapeutic agents. It also details the cellular and molecular mechanisms involved in chemosensitization.

Design: Search terms were combined and used to retrieve English language reports in PubMed, Science Direct and Scopus databases, published until October 2017. All articles were carefully analyzed and data extraction was conducted through standardized forms. Methodological quality assessment of in vivo studies was also performed.

Results: From a total of 669 articles surveyed, 104 met the inclusion criteria established. The main studied compounds as chemosensitizers were phenolic derivatives $(26.9 \%)$ and flavonoids $(17.3 \%)$. Most reports were authored by researchers from China (33.7\%) and USA (26.9\%). A large number of articles were published from 2011 to 2015 (50.0\%), suggesting that the use of natural products as chemosensitizers is a recent issue. In vivo studies were conducted mainly using xenograft models, which were considered of moderate methodological quality.

Conclusion: Several natural products, belonging to diverse chemical families, are potent chemosentisizers in tumor cells enhancing the cytotoxicity of conventional drugs. These molecules usually have a pleiotropic effect on different molecular targets, acting on several cellular and molecular processes with low selectivity. All studied molecules were obtained from terrestrial plants and major developments should arise from future studies, considering the chemodiversity of molecules purified from other terrestrial taxa and marine organisms.

Keywords: cancer; chemotherapy; chemosensitization; drug resistance; natural products 


\section{Introduction}

Cancer is one of the most impactful diseases of the $21^{\text {st }}$ century, affecting populations of diverse social, ethnic and economic characteristics. Although the genetic, epigenetic and pathophysiological mechanisms of cancer have been well described in recent years, cancer still represents the second cause of death in developed countries after heart disease $[1,2]$.

To ensure their survival and proliferation, cancer cells acquire differentiated abilities compared to normal cells. In the development of malignant tumors, they may present constitutively active proto-oncogenes, which predisposes to carcinogenesis, maintaining proliferative signaling pathways active [3]. In addition, expression of tumor suppressor genes is usually decreased and the cell acquires sufficient autonomy to continue multiplying without the need for growth factors. Tumor cells also have replicative immortality mechanisms [4] and greater resistance to cell death mediated by the regulation of anti and pro-apoptotic proteins [5]. For tumor maintenance and progression, they stimulate the production of angiogenic factors and modulate cellular metabolism in order to obtain more nutrients $[3,6]$.

In this sense, chemotherapy is one of the main alternatives for cancer treatment, using molecules capable of inhibiting proliferative signaling pathways, replicative immortality mechanisms and angiogenesis, besides inducing apoptosis of tumor cells [7-10]. However, the efficacy of conventional chemotherapeutics has been limited by drug resistance mechanisms [11]. Several studies have recognized that tumors exhibit a high degree of molecular and genetic heterogeneity, making them adapted to the usual cytotoxic agents. Unsuccessful treatments have been attributed to increased rates of drug efflux, alterations in drug metabolism (drug inhibition and degradation), cell death inhibition, epigenetic factor and mutations of drug targets (Figure 1). These mechanisms can act independently or in combination and through numerous signaling pathways [1113].

A wide variety of natural compounds has been reported for cancer therapy $[14,15]$. Natural products are an inexhaustible source of molecules with unique structural models and innovative mechanisms of action. In fact, natural compounds can be used in a versatile manner, especially in cancer management: a) as chemotherapeutic agents $[16,17]$; b) in cancer prevention (chemopreventive agents) $[18,19]$; c) or 
improving the effectiveness of conventional chemotherapy (chemosensitizer agents) [20].

Most of the identified chemosensitizer natural compounds are phytochemicals, which are classified as phenolic derivatives, flavonoids, alkaloids, carotenoids, terpenoids, quinones, saponins and steroids depending on their molecular structure $[20,21]$. In general, these molecules act by increasing the residence time of chemotherapeutics in tumor cells, inducing cell death by up-regulation of pro-apoptotic targets, promoting DNA damage or regulating the expression of altered and unaltered drug targets (Figure 1). When associated, these mechanisms enhance the cytotoxic effect of anticancer drugs, promoting a synergistic effect even in cells with acquired resistance [22-24].

The present systematic review was designed to summarize and analyze reports involving the use of natural products as chemosensitizers. Our focus was on preclinical studies (in vitro and in vivo approaches) in order to demonstrate to readers how these experimental models can contribute to the achievement of alternative strategies for cancer therapy.

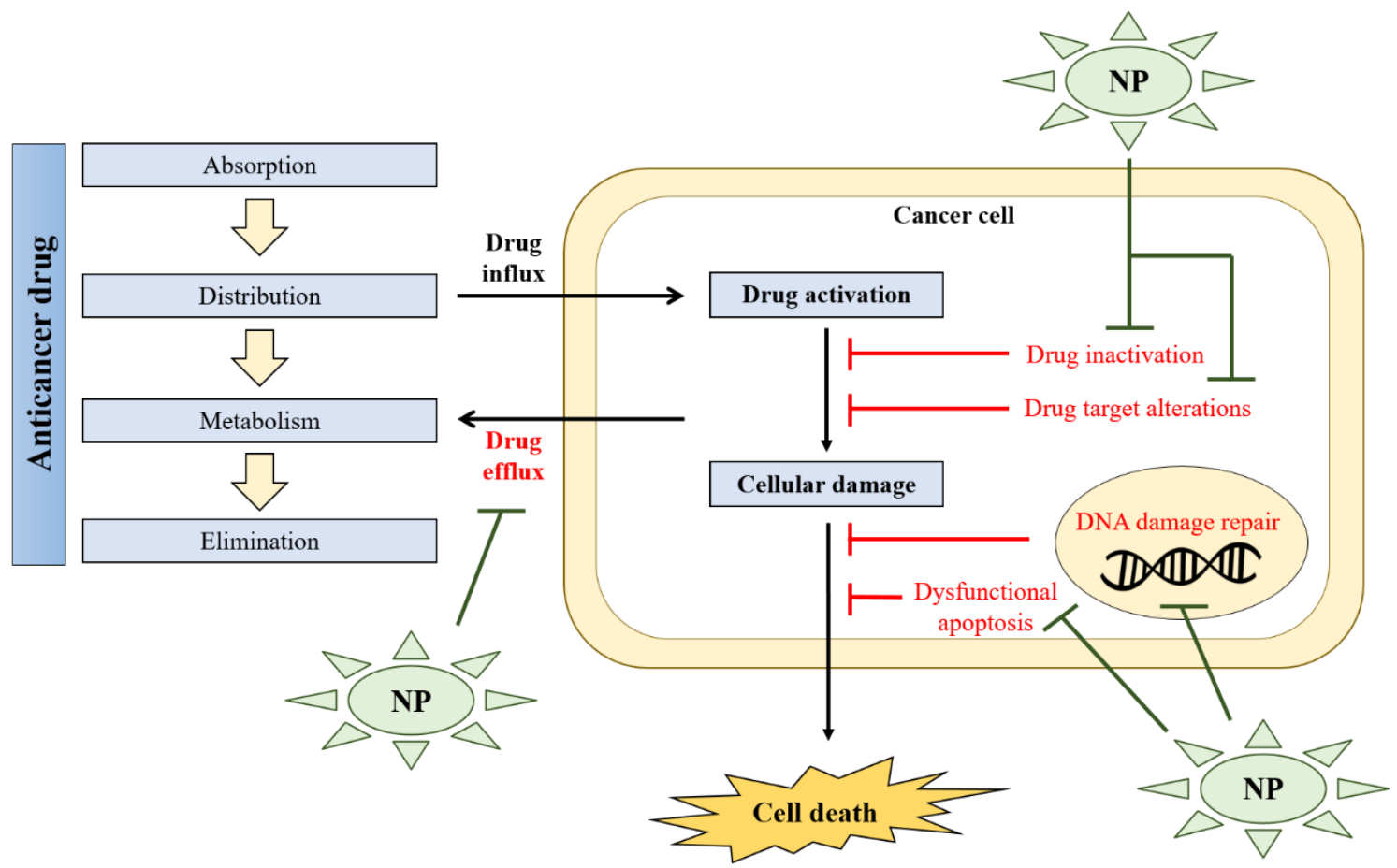

Figure 1. General drug resistance mechanisms implicated in cancer therapy and possibilities of intervention of natural products (NP) as chemosensitizer agents.

\section{Materials and methods}




\subsection{Search strategy}

A systematic review was conducted through a literature search performed in October 2017 and included all reports published to date. This literature search was performed on specialized databases (PubMed, Science Direct and Scopus) using different combinations of the following keywords: chemosensitization, cancer, tumor, natural products, phytotherapy, medicinal plants, marine products and marine drugs. We did not contact investigators and we did not attempt to identify unpublished data. This systematic review was performed in accordance with the criteria described on the Preferred Reporting Items for Systematic Reviews and Meta-Analyses (PRISMA) statement [25].

\subsection{Study selection}

Manuscript selection was based on the inclusion criteria: pre-clinical (in vitro and in vivo) studies involving the use of natural compounds/secondary metabolites as chemosensitizer agents of tumor cells to chemotherapeutic drugs, as well as pre-clinical (in vitro and in vivo) studies involving associations/combinatorial treatment between natural compounds/secondary metabolites and conventional chemotherapeutic drugs for antitumor therapy; only articles published in English and containing keywords in the title or abstract were selected. Other review articles, meta-analysis, abstracts, conferences, editorials/letters, case reports, conference proceedings, manuscripts without full text available or articles that did not meet the inclusion criteria were excluded from this systematic review. Studies involving extracts, fractions, synthetic or semisynthetic derivatives were also excluded.

For the selection of the manuscripts, two independent investigators (RGOJ and CAAF) first selected the articles according to the title, then to the abstract and finally through an analysis of the full-text publication. In cases of non-consensus, a third independent review was consulted (JRGSA). The selected articles were carefully reviewed with the purpose of identifying and excluding the reports that did not fit the criteria described above. Additional papers were included in this review after the analysis of all references from the selected articles. 


\subsection{Data extraction}

Data were collected and examined by the authors using standardized forms. The information from the selected manuscripts on studied natural compounds, experimental models, associated chemotherapeutic agent, doses or concentrations, route of administration, cell lines, biochemical assays, histological assessments and molecular mechanisms studied were extracted and assessed.

\subsection{Methodological quality assessment}

The risk of bias and quality of the in vivo preclinical investigations were assessed using a checklist adapted from Hooijmans et al. [26] and Siqueira-Lima et al. [27]. This analysis allowed evaluating the methodological quality of the selected studies regarding the randomization of the treatment allocation, blinded drug administration, blinded outcome assessment and outcome measurements. Studies that reported randomization of animals, blinding and outcome measurements were considered of higher methodological quality.

\section{Results}

The primary search identified 669 reports (08 from PubMed, 562 from Science Direct and 99 from Scopus). However, 147 manuscripts were indexed in two or more databases and were considered only once, resulting in 552 original articles. After an initial screening of titles and abstract, 436 articles were excluded since they did not meet the inclusion criteria or presented extremely different themes from the proposal of this systematic review. Finally, 86 articles were fully analyzed and among these 39 were excluded. A detailed analysis of the list of references from all selected articles was performed, leading to the addition of 57 papers pertinent to this review and that met all inclusion criteria established after title, abstract and full text analysis. In total, 104 articles were included for data extraction. A flowchart illustrating the progressive study selection and numbers at each stage is shown in Figure 2. 
Identified studies from the databases using keywords $(\mathrm{n}=669)$ : PubMed ( $\mathrm{n}=08)$, Science Direct $(\mathrm{n}=562)$, Scopus $(\mathrm{n}=99)$

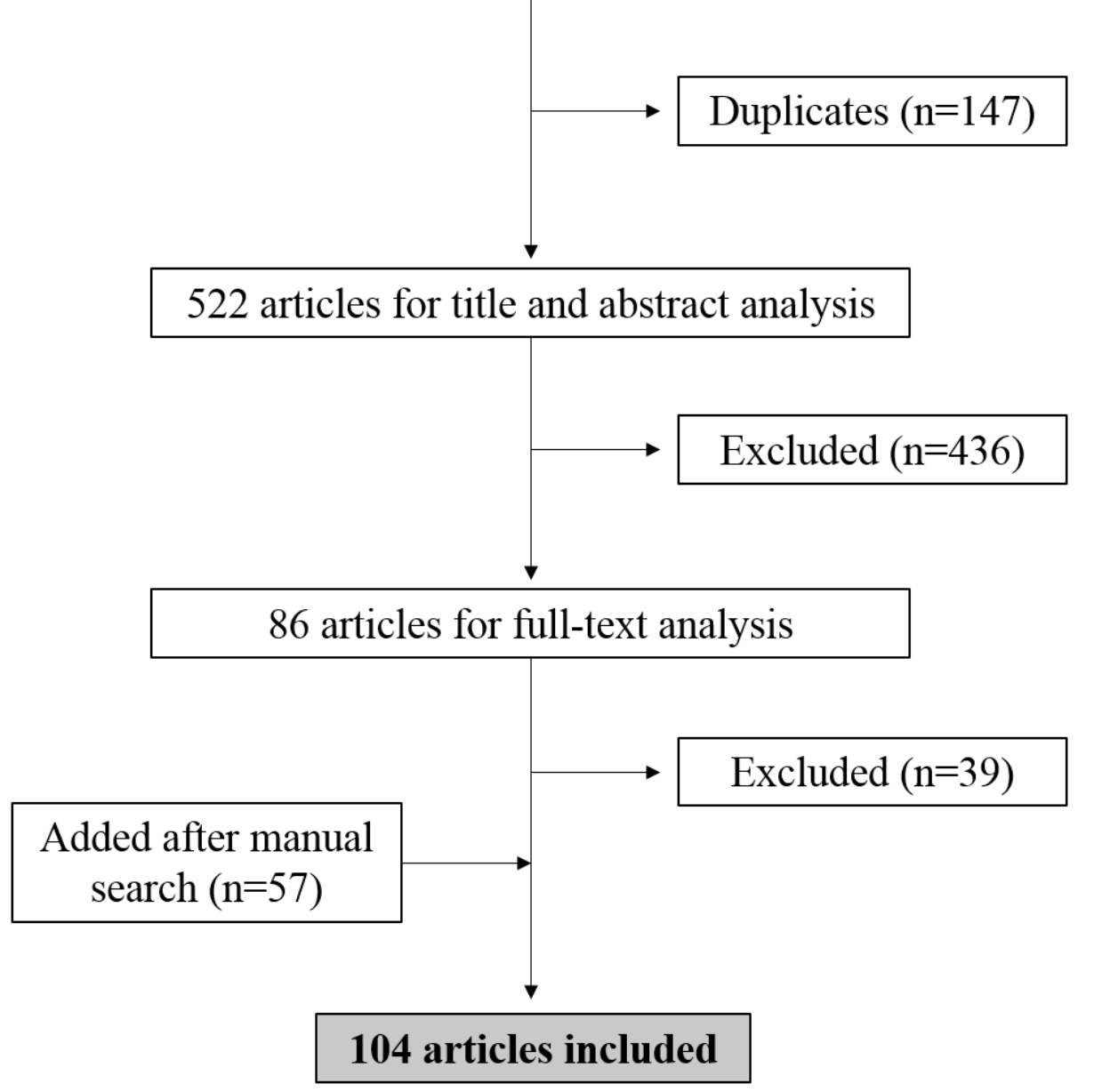

Figure 2. Flowchart detailing literature search according to PRISMA statement [25].

The articles selected for this review were categorically analyzed in relation to the country where the study was conducted, year of publication, natural compounds evaluated as chemosensitizers, cell lines and corresponding cancers. Table 1 summarizes the main informations contained in the selected in vitro and in vivo reports. In general, the studies were conducted by research groups located in about 20 different countries. However, most of the investigations were authored by researchers from China (35 reports, 33.7\%) and USA (28 reports, 26.9\%). Regarding the annual evolution of the publications, a large number of articles were published from 2011 to 2015 (52 reports, $50.0 \%)$. Only in the last two years, 18 articles (17.3\%) have been published, suggesting that the use of natural products as chemosensitizers is a recent issue that has attracted researchers' attention. 

Table 1. General characteristics of included studies (in vitro and in vivo reports).

\begin{tabular}{|c|c|c|c|c|c|}
\hline $\begin{array}{l}\text { Authors, year, } \\
\text { country }\end{array}$ & Model & Chemosensitizer & $\begin{array}{l}\text { Combined } \\
\text { conventional drug }\end{array}$ & Tumor cell line & Cancer \\
\hline \multicolumn{6}{|l|}{ Alkaloids } \\
\hline $\begin{array}{l}\text { Song et al. (2007) } \\
\text { [28], China }\end{array}$ & $\begin{array}{l}\text { In vitro and } \\
\text { in vivo }\end{array}$ & Oxymatrine & NM-3 & $\begin{array}{l}\text { SGC-7901, MKN-45 and } \\
\text { MKN-74 }\end{array}$ & Gastric cancer \\
\hline $\begin{array}{l}\text { Banerjee et al. } \\
(2009) \text { [29], USA }\end{array}$ & $\begin{array}{l}\text { In vitro and } \\
\text { in vivo }\end{array}$ & 3,3-diindolylmethane & $\begin{array}{l}\text { CIP, OXP and } \\
\text { GCT }\end{array}$ & $\begin{array}{l}\text { PANC-1, Colo-357 and } \\
\text { PANC- } 28\end{array}$ & Pancreatic cancer \\
\hline $\begin{array}{l}\text { Sung et al. (2010) } \\
\text { [30], USA }\end{array}$ & In vitro & Noscapine & $\begin{array}{l}\text { TNF, TLD, PTX } \\
\text { and BTZ }\end{array}$ & KBM-5 and U266 & Leukemia \\
\hline $\begin{array}{l}\text { Chougule et al. } \\
\text { (2011) [31], USA }\end{array}$ & $\begin{array}{l}\text { In vitro and } \\
\text { in vivo }\end{array}$ & Noscapine & GCT & A549 and H460 & Lung cancer \\
\hline $\begin{array}{l}\text { Tong et al. (2012a) } \\
\text { [32], China }\end{array}$ & In vitro & Berberine & DOX & A549; HeLa; HepG2 & $\begin{array}{l}\text { Lung cancer; cervical } \\
\text { cancer; hepatocellular } \\
\text { carcinoma }\end{array}$ \\
\hline $\begin{array}{l}\text { Qi et al. (2013) [33], } \\
\text { USA }\end{array}$ & $\begin{array}{l}\text { In vitro and } \\
\text { in vivo }\end{array}$ & Noscapine & $\begin{array}{l}\text { TMZ, BCE and } \\
\text { CIP }\end{array}$ & U87MG & Glioblastoma \\
\hline $\begin{array}{l}\text { Wang et al. (2013a) } \\
\text { [34], China }\end{array}$ & $\begin{array}{l}\text { In vitro and } \\
\text { in vivo }\end{array}$ & Sinomenine & $5 \mathrm{FU}$ & Eca-109 & Esophageal carcinoma \\
\hline $\begin{array}{l}\text { Guo et al. (2014) } \\
\text { [35]. China }\end{array}$ & In vitro & Berberine & RPM & SMMC7721 and HepG2 & Hepatocellular carcinoma \\
\hline
\end{tabular}


Liu et al. (2015a)

[36], China

Doddapaneni et al.

(2016) [37], USA

Zhao et al. (2016)

[38], China

\section{Carotenoids}

Rajendran et al.

(2010) [39],

In vitro

$\gamma$-Tocotrienol

Singapore

Liu et al. (2015b)

[40], China

Zhang et al. (2016)

[41], China

In vitro and Oxymatrine

in vivo

In vitro

In vitro

Berberine

Invitro and

$\alpha$-carotene

in vivo

In vitro and $\quad \beta$-carotene

in vivo

Coumarins

Kim et al. (2014)

In vitro Bergamottin

BTZ and TLD

U266

Multiple myeloma
CIP

DOX and PTX

Hep-G2 and SMMC7721

MDA-MB231

MCF-7

HepG2, C3A, SNU-387, and PLC/PRF5

PTX

$\mathrm{LLC}^{* * *}$

EC1 and Eca109

Esophageal carcinoma
Breast cancer

Breast cancer

[42], South Korea

\section{Flavonoids}

Stammler and Volm In vitro

(1997) [43],

Germany
Epigallocatechin-3- DOX gallate 


\begin{tabular}{|c|c|c|c|c|c|}
\hline $\begin{array}{l}\text { Dhanalakshmi et al. } \\
\text { (2003) [44], USA }\end{array}$ & In vitro & Silibinin & CIP and CAP & DU145 & Prostate cancer \\
\hline $\begin{array}{l}\text { Chisholm et al. } \\
(2004) \text { [45], New } \\
\text { Zealand }\end{array}$ & In vitro & $\begin{array}{l}\text { Epigallocatechin-3- } \\
\text { gallate }\end{array}$ & TOF & MDA-MB-231 & Breast cancer \\
\hline $\begin{array}{l}\text { Peng et al. (2007) } \\
\text { [46], USA }\end{array}$ & In vitro & Deguelin & DOX and DTX & $\begin{array}{l}\text { SKBR-3, MCF-7 and } \\
\text { MCF } 10 A\end{array}$ & Breast cancer \\
\hline $\begin{array}{l}\text { Siddiqui et al. (2008) } \\
\text { [47], USA }\end{array}$ & In vitro & $\begin{array}{l}\text { Epigallocatechin-3- } \\
\text { gallate }\end{array}$ & TRAIL & $\mathrm{LNCaP}$ & Prostate cancer \\
\hline $\begin{array}{l}\text { Shervington et al. } \\
\text { (2009) [48], UK }\end{array}$ & In vitro & $\begin{array}{l}\text { Epigallocatechin-3- } \\
\text { gallate }\end{array}$ & CIP and TOF & $1321 \mathrm{~N} 1 ; \mathrm{U} 87-\mathrm{MG}$ & Astrocytoma; glioblastoma \\
\hline $\begin{array}{l}\text { Zhang et al. (2009) } \\
\text { [49], China }\end{array}$ & $\begin{array}{l}\text { In vitro and } \\
\text { in vivo }\end{array}$ & Naringenin & DOX & $\begin{array}{l}\text { A549; HepG2; MCF-7 } \\
\text { and MCF-7/DOX }\end{array}$ & $\begin{array}{l}\text { Lung cancer; hepatocellular } \\
\text { carcinoma; breast cancer }\end{array}$ \\
\hline $\begin{array}{l}\text { Jin et al. (2011) [50], } \\
\text { South Korea }\end{array}$ & In vitro & Naringenin & TRAIL & A549 & Lung cancer \\
\hline $\begin{array}{l}\text { Stearns and Wang } \\
\text { (2011) [51], USA }\end{array}$ & $\begin{array}{l}\text { In vitro and } \\
\text { in vivo }\end{array}$ & $\begin{array}{l}\text { Epigallocatechin-3- } \\
\text { gallate }\end{array}$ & TXN & PC-3ML & Prostate cancer \\
\hline $\begin{array}{l}\text { Hönicke et al. (2012) } \\
\text { [52], Germany }\end{array}$ & In vitro & $\begin{array}{l}\text { Epigallocatechin-3- } \\
\text { gallate }\end{array}$ & IL-1Ra & $\mathrm{U}-2 \mathrm{OS}$ & Osteosarcoma \\
\hline $\begin{array}{l}\text { Wu et al. (2012) } \\
\text { [53], China }\end{array}$ & In vivo & $\begin{array}{l}\text { Epigallocatechin-3- } \\
\text { gallate }\end{array}$ & $\mathrm{CCT}$ & BGC-823 & Gastric cancer \\
\hline
\end{tabular}




\begin{tabular}{|c|c|c|c|c|c|}
\hline $\begin{array}{l}\text { Kwak et al. (2013) } \\
\text { [54], South Korea }\end{array}$ & In vitro & $\begin{array}{l}\text { Epigallocatechin-3- } \\
\text { gallate }\end{array}$ & VOR & HuCC-T1 & Cholangiocarcinoma \\
\hline $\begin{array}{l}\text { Suzuki et al. (2014) } \\
\text { [55], USA }\end{array}$ & $\begin{array}{l}\text { In vitro and } \\
\text { in vivo }\end{array}$ & Genistein & $5 \mathrm{FU}$ & MIA PaCa-2 & Pancreatic cancer \\
\hline $\begin{array}{l}\text { Wang et al. (2014a) } \\
\text { [56], China }\end{array}$ & $\begin{array}{l}\text { In vitro and } \\
\text { in vivo }\end{array}$ & Myricetin & $5 \mathrm{FU}$ & EC9706 & Esophageal carcinoma \\
\hline $\begin{array}{l}\text { Abaza et al. (2015) } \\
\text { [57], Kuwait }\end{array}$ & In vitro & Naringenin & $\begin{array}{l}\text { CPT, 5FU, DOX, } \\
\text { CIP, ELP, ETP, } \\
\text { CAP and CPA }\end{array}$ & $\begin{array}{l}\text { SW1116 and SW837; } \\
\text { HTB26 and HTB132 }\end{array}$ & Colon cancer; breast cancer \\
\hline $\begin{array}{l}\text { Wang et al. (2015) } \\
\text { [58], USA }\end{array}$ & In vitro & $\begin{array}{l}\text { Epigallocatechin-3- } \\
\text { gallate + quercetin }\end{array}$ & DTX & LAPC-4-AI and PC-3 & Prostate cancer \\
\hline $\begin{array}{l}\text { García-Vilas et al. } \\
\text { (2016) [59], Spain }\end{array}$ & In vitro & $\begin{array}{l}\text { Epigallocatechin-3- } \\
\text { gallate }\end{array}$ & $4 \mathrm{MU}$ & MDA-MB231 & Breast cancer \\
\hline $\begin{array}{l}\text { Krajnovic et al. } \\
(2016) \text { [60], Serbia }\end{array}$ & $\begin{array}{l}\text { In vitro and } \\
\text { in vivo }\end{array}$ & Isoxanthohumol & PTX & $\mathrm{B} 16, \mathrm{~A} 375$ and $\mathrm{B} 16 \mathrm{~F} 10$ & Melanoma \\
\hline \multicolumn{6}{|l|}{ Naphthodianthrones } \\
\hline $\begin{array}{l}\text { Lin et al. (2016) } \\
\text { [61], China }\end{array}$ & In vitro & Hypericin & OXA & HCT8 and HCT116 & Colon cancer \\
\hline $\begin{array}{l}\text { Lin et al. (2017) } \\
\text { [62], China }\end{array}$ & In vitro & Hypericin & OXP & HCT116 and HCT8 & Colon cancer \\
\hline
\end{tabular}

Phenolic derivatives 


\begin{tabular}{|c|c|c|c|c|c|}
\hline $\begin{array}{l}\text { Anuchapreeda et al. } \\
\text { (2002) [63], } \\
\text { Thailand }\end{array}$ & In vitro & Curcumin & VBL & KB-V1 & Cervical cancer \\
\hline $\begin{array}{l}\text { Hour et al. (2002) } \\
\text { [64], China }\end{array}$ & In vitro & Curcumin & $\begin{array}{l}\text { DOX, } 5 \mathrm{FU} \text { and } \\
\text { PTX }\end{array}$ & PC-3 and DU145 & Prostate cancer \\
\hline $\begin{array}{l}\text { Fulda and Debatin } \\
(2004)[65], \\
\text { Germany }\end{array}$ & In vitro & Resveratrol & $\begin{array}{l}\text { DOX, VP16, ACD, } \\
\text { PTX, MET, CYT, } \\
\text { 5FU, CHM, MMS, } \\
\text { TMD and NCD }\end{array}$ & $\begin{array}{l}\text { SHEP; U373MG; } \\
\text { PANC1; MCF7; LNCaP; } \\
\text { Jurkat T-cell and Reh B- } \\
\text { cell }\end{array}$ & $\begin{array}{l}\text { Neuroblastoma; malignant } \\
\text { glioma; pancreatic cancer; } \\
\text { breast cancer; prostate } \\
\text { cancer; leukemia }\end{array}$ \\
\hline $\begin{array}{l}\text { Wu et al. (2004) } \\
\text { [66], China }\end{array}$ & In vivo & Resveratrol & $5 \mathrm{FU}$ & $\mathrm{H}_{22}$ & Hepatocellular carcinoma \\
\hline $\begin{array}{l}\text { Aggarwal et al. } \\
\text { (2005) [67], USA }\end{array}$ & $\begin{array}{l}\text { In vitro and } \\
\text { in vivo }\end{array}$ & Curcumin & PTX & MDA-MB-435 & Breast cancer \\
\hline $\begin{array}{l}\text { Bava et al. (2005) } \\
\text { [68], India }\end{array}$ & In vitro & Curcumin & PTX & $\begin{array}{l}\text { HeLa, SiHa, CaSki, and } \\
\text { ME-180 }\end{array}$ & Cervical cancer \\
\hline $\begin{array}{l}\text { Li et al. (2007) [69], } \\
\text { USA }\end{array}$ & $\begin{array}{l}\text { In vitro and } \\
\text { in vivo }\end{array}$ & Curcumin & OXA & LoVo and Colo205 & Colon cancer \\
\hline $\begin{array}{l}\text { Chen et al. (2009) } \\
\text { [70], Taiwan }\end{array}$ & In vitro & Tannic acid & ATO & HL-60 & Leukemia \\
\hline $\begin{array}{l}\text { Harikumar et al. } \\
\text { (2009) [71], USA }\end{array}$ & $\begin{array}{l}\text { In vitro and } \\
\text { in vivo }\end{array}$ & Resveratrol & GCT & $\begin{array}{l}\text { AsPC-1, MIA PaCa-2 } \\
\text { and PANC1 }\end{array}$ & Pancreatic cancer \\
\hline Kunnumakkara et al. & In vitro and & Curcumin & $\mathrm{CCT}$ & HCT116, HT29 and & Colon cancer \\
\hline
\end{tabular}




\begin{tabular}{|c|c|c|c|c|c|}
\hline (2009) [72], USA & in vivo & & & SW620 & \\
\hline $\begin{array}{l}\text { Yu et al. (2009) [73], } \\
\text { USA }\end{array}$ & In vitro & Curcumin & FOLFOX & HCT116 and HT29 & Colon cancer \\
\hline $\begin{array}{l}\text { Hartojo et al. (2010) } \\
\text { [74], USA }\end{array}$ & In vitro & Curcumin & 5FU and CIP & Flo-1 and OE33 & Esophageal adenocarcinoma \\
\hline $\begin{array}{l}\text { Bava et al. (2011) } \\
\text { [75], India }\end{array}$ & In vitro & Curcumin & PTX & HeLa & Cervical cancer \\
\hline $\begin{array}{l}\text { Sreekanth et al. } \\
\text { (2011) [76], India }\end{array}$ & $\begin{array}{l}\text { In vitro and } \\
\text { in vivo }\end{array}$ & Curcumin & PTX & 3-MC* & Cervical cancer \\
\hline $\begin{array}{l}\text { Osman et al. (2012) } \\
\text { [77], Saudi Arabia }\end{array}$ & In vitro & Resveratrol & DOX & MCF-7 & Breast cancer \\
\hline $\begin{array}{l}\text { Saleh et al. (2012) } \\
\text { [78], Egypt }\end{array}$ & In vitro & Curcumin & ETP & $\begin{array}{l}\text { MCF-7; HeLa; HCT116; } \\
\text { HepG2; U251 }\end{array}$ & $\begin{array}{l}\text { Breast cancer; cervical } \\
\text { cancer; colon cancer; } \\
\text { hepatocellular carcinoma; } \\
\text { glioblastoma }\end{array}$ \\
\hline $\begin{array}{l}\text { Wang et al. (2012a) } \\
\text { [79], China }\end{array}$ & In vitro & Curcumin & LAP & RS4;11, Reh and Jurkat & $\begin{array}{l}\text { Acute lymphoblastic } \\
\text { leukemia }\end{array}$ \\
\hline $\begin{array}{l}\text { Amiri et al. (2013) } \\
\text { [80], Iran }\end{array}$ & In vitro & Resveratrol & ETP & HepG2; HCT116 & $\begin{array}{l}\text { Hepatocellular carcinoma; } \\
\text { colon cancer }\end{array}$ \\
\hline $\begin{array}{l}\text { Díaz-Chávez et al. } \\
\text { (2013) [81], Mexico }\end{array}$ & In vitro & Resveratrol & DOX & MCF-7 & Breast cancer \\
\hline Shakibaei et al. & In vitro & Curcumin & $5 \mathrm{FU}$ & HCT116 and & Colon cancer \\
\hline
\end{tabular}


(2013) [82],

Germany

Carlson et al. (2014) In vitro
[83], USA

Curcumin + resveratrol DOX

Qian et al. (2014)

[84], China

Buhrmann et al. In vitro Resveratrol

(2015) [85],

Germany

Cote et al. (2015)

[86], USA

Shakibaei et al.

(2015) [87],

Germany

Abaza et al. (2016) In vitro Methylferulate

[88], Kuwait

In vitro $\quad$ Resveratrol + quercetin

Ooko et al. (2016)

In vitro

Curcumin

[89], Germany

Tyagi et al. (2017)

In vitro

[90], USA
ADM

DOX

$5 \mathrm{FU}$

CPT, 5FU, DOX OXP, PTX, VBL,

VCR, ETP, ELP,

AMS, HHG and

APD

DOX

HCT116+ch3

SKOV-3 Ovarian cancer

HepG2 Hepatocellular carcinoma

HCT116, HCT116R, Colon cancer SW480 and SW480R

SKOV-3

Ovarian cancer

HCT116 and HCT116R Colon cancer

SW1116 and SW837 Colon cancer

CCRF-CEM and CEM/ADR5000

Acute lymphoblastic

leukemia

5FU and TLD $\quad \mathrm{KBM}-5$ 


\begin{tabular}{|c|c|c|c|c|c|}
\hline $\begin{array}{l}\text { Jafri et al. (2010) } \\
\text { [91], USA }\end{array}$ & $\begin{array}{l}\text { In vitro and } \\
\text { in vivo }\end{array}$ & Thymoquinone & CIP & $\begin{array}{l}\text { NCI-H460 and NCI- } \\
\text { H146 }\end{array}$ & Lung cancer \\
\hline $\begin{array}{l}\text { Li et al. (2010) [92], } \\
\text { Singapore }\end{array}$ & In vitro & Thymoquinone & BTZ and TLD & U266 and RPMI 8226 & Multiple myeloma \\
\hline $\begin{array}{l}\text { Sandur et al. (2010) } \\
\text { [93], USA }\end{array}$ & In vitro & Plumbagin & BTZ and TLD & U266 and MM.1S & Multiple myeloma \\
\hline $\begin{array}{l}\text { Effenberger- } \\
\text { Neidnicht and } \\
\text { Schobert (2011) } \\
\text { [94], Germany }\end{array}$ & In vitro & Thymoquinone & DOX & $\begin{array}{l}\text { HL-60; 518A2; HT-29; } \\
\text { KB-V1; MCF-7 }\end{array}$ & $\begin{array}{l}\text { Leukemia; melanoma; colon } \\
\text { cancer; cervical cancer; } \\
\text { breast cancer }\end{array}$ \\
\hline $\begin{array}{l}\text { Wang et al. (2014b) } \\
\text { [95], China }\end{array}$ & $\begin{array}{l}\text { In vitro and } \\
\text { in vivo }\end{array}$ & Shikonin & GCT & $\begin{array}{l}\text { PANC-1, BxPC-3 and } \\
\text { AsPC-1 }\end{array}$ & Pancreatic cancer \\
\hline $\begin{array}{l}\text { Daqian et al. (2015) } \\
\text { [96], China }\end{array}$ & In vitro & Chimaphilin & DOX & U-2OS and U-2OSMR & Osteosarcoma \\
\hline $\begin{array}{l}\text { He et al. (2016) [97], } \\
\text { China }\end{array}$ & $\begin{array}{l}\text { In vitro and } \\
\text { in vivo }\end{array}$ & Shikonin & CIP & $\begin{array}{l}\text { HCT116, HT29 and } \\
\text { SW620 }\end{array}$ & Colon cancer \\
\hline $\begin{array}{l}\text { Song et al. (2016) } \\
\text { [98], China }\end{array}$ & $\begin{array}{l}\text { In vitro and } \\
\text { in vivo }\end{array}$ & Shikonin & ATO & HepG2, Hep3B, Huh7 & Hepatocellular carcinoma \\
\hline $\begin{array}{l}\text { Wang et al. (2017) } \\
\text { [99], China }\end{array}$ & In vitro & Cryptotanshinone & PTX & CAL 27 and SCC 9 & $\begin{array}{l}\text { Tongue squamous cell } \\
\text { carcinoma }\end{array}$ \\
\hline
\end{tabular}




\section{Saponins}

\begin{tabular}{|c|c|c|c|c|c|}
\hline $\begin{array}{l}\text { Choi et al. (2003) } \\
\text { [100], South Korea }\end{array}$ & In vitro & Protopanaxatriol & DOX & $\begin{array}{l}\text { AML-2/D100 and AML- } \\
\text { 2/DX100 }\end{array}$ & Acute myeloid leukemia \\
\hline $\begin{array}{l}\text { Kim et al. (2010) } \\
\text { [101], South Korea }\end{array}$ & In vitro & Ginsenoside $\operatorname{Rg} 3$ & DTX & $\begin{array}{l}\text { LNCaP, PC-3 and } \\
\text { DU145 }\end{array}$ & Prostate cancer \\
\hline $\begin{array}{l}\text { Ming et al. (2010) } \\
\text { [102], China }\end{array}$ & In vitro & $\beta$-aescin & $5 \mathrm{FU}$ & SMMC-7721 & Hepatocellular carcinoma \\
\hline $\begin{array}{l}\text { Wang et al. (2012b) } \\
\text { [103], China }\end{array}$ & $\begin{array}{l}\text { In vitro and } \\
\text { in vivo }\end{array}$ & Escin & GCT & $\begin{array}{l}\text { L6, BxPC-3, PANC-1, } \\
\text { CFPAC-1 and SW-1990 }\end{array}$ & Pancreatic cancer \\
\hline $\begin{array}{l}\text { Yang et al. (2012) } \\
\text { [104], China }\end{array}$ & In vivo & Ginsenoside Rg3 & PTX & MCF-7 & Breast cancer \\
\hline $\begin{array}{l}\text { Wang et al. (2013b) } \\
\text { [105], China }\end{array}$ & $\begin{array}{l}\text { In vitro and } \\
\text { in vivo }\end{array}$ & Steroidal saponin & $\begin{array}{l}\text { DOX, 5FU, PTX } \\
\text { and CIP }\end{array}$ & HepG2 and R-HepG2 & Hepatocellular carcinoma \\
\hline $\begin{array}{l}\text { Chang et al. (2014) } \\
\text { [106], China }\end{array}$ & In vivo & Ginsenoside Rg3 & PTX + CIP & Eca- 109 & Esophageal carcinoma \\
\hline $\begin{array}{l}\text { Lee et al. (2014) } \\
\text { [107], South Korea }\end{array}$ & In vitro & Ginsenoside Rg3 & CIP & $\begin{array}{l}\text { HTB5, J82, JON, } \\
\text { UMUC14 and T24 }\end{array}$ & Bladder cancer \\
\hline $\begin{array}{l}\text { Liu et al. (2017) } \\
\text { [108], China }\end{array}$ & In vitro & Paris saponin I & $\mathrm{CPT}$ & $\begin{array}{l}\text { H1299; H520; H460; } \\
\text { H446 }\end{array}$ & $\begin{array}{l}\text { Lung adenocarcinoma; lung } \\
\text { squamous cell carcinoma; } \\
\text { lung large cell carcinoma }\end{array}$ \\
\hline
\end{tabular}




\begin{tabular}{|c|c|c|c|c|c|}
\hline $\begin{array}{l}\text { Yuan et al. (2017) } \\
\text { [109], China }\end{array}$ & $\begin{array}{l}\text { In vitro and } \\
\text { in vivo }\end{array}$ & Ginsenoside Rg3 & PTX & $\begin{array}{l}\text { MDA-MB-231, } \\
\text { MDA-MB-453 and BT- } \\
549\end{array}$ & Breast cancer \\
\hline \multicolumn{6}{|l|}{ Steroids } \\
\hline $\begin{array}{l}\text { Lee et al. (2009) } \\
\text { [110], South Korea }\end{array}$ & In vitro & Withaferin A & TRAIL & $\begin{array}{l}\text { Caki, Huh7, SK-Hep1 } \\
\text { and Hep3B }\end{array}$ & Renal cancer \\
\hline $\begin{array}{l}\text { Chen et al. (2010) } \\
\text { [111], South Africa }\end{array}$ & In vitro & Cucurbitacin B & CIP & $\begin{array}{l}\text { SRB1, SRB12, SCC13 } \\
\text { and COLO160 }\end{array}$ & $\begin{array}{l}\text { Cutaneous squamous } \\
\text { carcinoma }\end{array}$ \\
\hline $\begin{array}{l}\text { Iwanski et al. (2010) } \\
\text { [112], USA }\end{array}$ & In vivo & Cucurbitacin B & GCT & PANC-1 & Pancreatic cancer \\
\hline $\begin{array}{l}\text { Lee et al. (2011) } \\
\text { [113], USA }\end{array}$ & $\begin{array}{l}\text { In vitro and } \\
\text { in vivo }\end{array}$ & Cucurbitacin B & MET & $\begin{array}{l}\text { U2OS, G292, MG-63, } \\
\text { HT-161, HOS, SAOS-2, } \\
\text { and SJSA }\end{array}$ & Osteosarcoma \\
\hline $\begin{array}{l}\text { Cohen et al. (2012) } \\
\text { [114], USA }\end{array}$ & In vitro & Withaferin A & SOF & BCPAP and SW1736 & Thyroid cancer \\
\hline $\begin{array}{l}\text { Fong et al. (2012) } \\
{[115], \text { USA }}\end{array}$ & $\begin{array}{l}\text { In vitro and } \\
\text { in vivo }\end{array}$ & Withaferin A & DOX & $\begin{array}{l}\mathrm{A} 2780, \mathrm{~A} 2780 / \mathrm{CP} 70 \text { and } \\
\mathrm{CaOV} 3\end{array}$ & Ovarian cancer \\
\hline $\begin{array}{l}\text { El-Senduny et al. } \\
\text { (2015) [116], USA }\end{array}$ & In vitro & Cucurbitacin B & CIP & $\mathrm{A} 2780$ and $\mathrm{A} 2780 \mathrm{CP}$ & Ovarian cancer \\
\hline $\begin{array}{l}\text { Li et al. (2015) } \\
\text { [117], China }\end{array}$ & $\begin{array}{l}\text { In vitro and } \\
\text { in vivo }\end{array}$ & Withaferin A & OXP & $\begin{array}{l}\text { PANC-1, MIAPaCa-2 } \\
\text { and SW1990 }\end{array}$ & Pancreatic cancer \\
\hline
\end{tabular}




\begin{tabular}{|c|c|c|c|c|c|}
\hline $\begin{array}{l}\text { Ben-Eltriki et al. } \\
\text { (2016) [118], } \\
\text { Canada }\end{array}$ & In vitro & $20(S)$-protopanaxadiol & CAL & LNCaP and C4-2 & Prostate cancer \\
\hline \multicolumn{6}{|l|}{ Terpenoids } \\
\hline $\begin{array}{l}\text { Holland et al. (2006) } \\
\text { [119], Australia }\end{array}$ & In vitro & $\begin{array}{l}\text { Cannabidiol, } \Delta^{9}- \\
\text { tetrahydrocannabinol }\end{array}$ & VBL & $\begin{array}{l}\text { CCRF-CEM and } \\
\text { CEM/VLB } 100\end{array}$ & $\begin{array}{l}\text { Acute T-lymphoblastoid } \\
\text { leukemia }\end{array}$ \\
\hline $\begin{array}{l}\text { Sieber et al. (2009) } \\
{[120], \text { Germany }}\end{array}$ & In vitro & Artesunate & RUX & Ramos & B-lymphoma \\
\hline $\begin{array}{l}\text { He et al. (2011) } \\
\text { [121], China }\end{array}$ & In vitro & Lupeol & TRAIL & SMMC7721 and HepG2 & Hepatocellular carcinoma \\
\hline $\begin{array}{l}\text { Kannaiyan et al. } \\
\text { (2011) [122], } \\
\text { Singapore }\end{array}$ & In vitro & Celastrol & TLD and BTZ & $\begin{array}{l}\text { U266, RPMI 8226, } \\
\text { RPMI-8226 and RPMI- } \\
\text { 8226-LR-5 }\end{array}$ & Multiple myeloma \\
\hline $\begin{array}{l}\text { Torres et al. (2011) } \\
\text { [123], Spain }\end{array}$ & $\begin{array}{l}\text { In vitro and } \\
\text { in vivo }\end{array}$ & $\begin{array}{l}\text { Cannabidiol and } \Delta^{9}- \\
\text { tetrahydrocannabinol }\end{array}$ & TMZ & $\begin{array}{l}\text { U87MG (U87), A172, } \\
\text { SW1783, U373MG } \\
\text { (U373), T98G (T98), } \\
\text { SW1088, and LN405 }\end{array}$ & Glioma \\
\hline $\begin{array}{l}\text { Prasad et al. (2012) } \\
\text { [124], USA }\end{array}$ & In vivo & Ursolic acid & $\mathrm{CCT}$ & $\begin{array}{l}\text { HCT116, HT29, and } \\
\text { Caco2 }\end{array}$ & Colon cancer \\
\hline $\begin{array}{l}\text { Tong et al. (2012b) } \\
\text { [125], China }\end{array}$ & In vitro & Pseudolaric acid B & $\mathrm{ABT}-737 * * *$ & $\begin{array}{l}\text { LNCaP, PC-3 and } \\
\text { DU145 }\end{array}$ & Prostate cancer \\
\hline Wang et al. (2012c) & In vitro & Pristimerin & GCT & BxPC-3, PANC-1 and & Pancreatic cancer \\
\hline
\end{tabular}




\begin{tabular}{|c|c|c|c|c|c|}
\hline $\begin{array}{l}\text { Butturini et al. } \\
\text { (2013) [127], Italy }\end{array}$ & In vitro & Cynaropicrin & CIP and DTX & THP-1 & Monocytic leukemia \\
\hline $\begin{array}{l}\text { Liu et al. (2013) } \\
\text { [128], China }\end{array}$ & $\begin{array}{l}\text { In vitro and } \\
\text { in vivo }\end{array}$ & Lupeol & S14161 & HepG2 and SMMC7721 & Hepatocellular carcinoma \\
\hline $\begin{array}{l}\text { Bamodu et al. (2015) } \\
\text { [129], Taiwan }\end{array}$ & In vitro & Ovatodiolide & DOX & $\begin{array}{l}\text { MDA-MB-231, HS578T } \\
\text { and MCF-7 }\end{array}$ & Breast cancer \\
\hline $\begin{array}{l}\text { Liu et al. (2016) } \\
\text { [130], China }\end{array}$ & $\begin{array}{l}\text { In vitro and } \\
\text { in vivo }\end{array}$ & Lupeol & $5 \mathrm{FU}$ & SGC7901 and BGC823 & Gastric carcinoma \\
\hline $\begin{array}{l}\text { Reis et al. (2016) } \\
{[131], \text { Portugal }}\end{array}$ & In vitro & $\begin{array}{l}\text { Euphowelwitschines A } \\
\text { and B, welwitschene, } \\
\text { epoxywelwitschene and } \\
\text { esulatin M }\end{array}$ & DOX & L5178Y & T-lymphoma \\
\hline
\end{tabular}

4-methylumbelliferone (4MU); 5-fluorouracil (5FU); adriamycin (ADM); amsacrine (AMS); actinomycin D (ACD); aphidicolin (APD); arsenic trioxide (ATO); bis-chloroethylnitrosourea (BCE); bortezomib (BTZ); calcitrol (CAL); camptothecin (CPT); capecitabine (CCT); carboplatin (CAP); cisplatin (CIP); cyclophosphamide (CPA); cycloheximide (CHM); cytarabine (CYT); docetaxel (DTX); doxorubicin (DOX); ellipticine (ELP); etopside (ETP); 5FU + oxilaplatin (FOLFOX); gemcitabine (GCT); homoharrigtonine (HHG); ifosfamide (IFO); IL-1 receptor antagonist (IL-Ra); L-asparaginase (LAP); methotrexate (MET); mimosine (MMS); nocodazole (NCD); oxaliplatin (OXP); paclitaxel (PTX); rapamycin (RPM); rituximab (RUX); sorafenib (SOF); tamoxifen (TOF); taxane (TXN): DTX + PTX; temozolamide (TMZ); thalidomide (TLD); thymidine (TMD); TNF-Related Apoptosis Inducing Ligand (TRAIL); vinblastine (VBL); vincristine (VCR); vorinostat (VOR). *3-Methylcholanthrene (3MC)-induced tumorigenesis in Swiss albino mice. **LLC: mouse Lewis lung cancer cell line. ***ABT-737: Bcl-2 inhibitor. 

Combinatorial therapy (natural compounds and conventional chemotherapeutic) were used in various types of cancer. Breast and colon cancer were the most cited (16 reports each), followed by leukemia and associate cancers, lung, pancreatic, prostate and cervical cancer. Concerning the conventional anticancer drugs mentioned, about 40 different chemotherapeutic agents have been reported in combination with one or more natural molecules, varying according to the type of cancer studied, as shown in Table 1. Similarly, a wide variety of natural compounds have been reported as chemosensitizer agents. Most of the molecules studied belong to the class of phenolic derivatives (28 reports, $26.9 \%$ ) and flavonoids (18 reports, 17.3\%). Besides these, terpenoids, alkaloids, saponins, quinones and steroids were also considerably cited. These and other important outcomes are graphically presented in figure 3 .
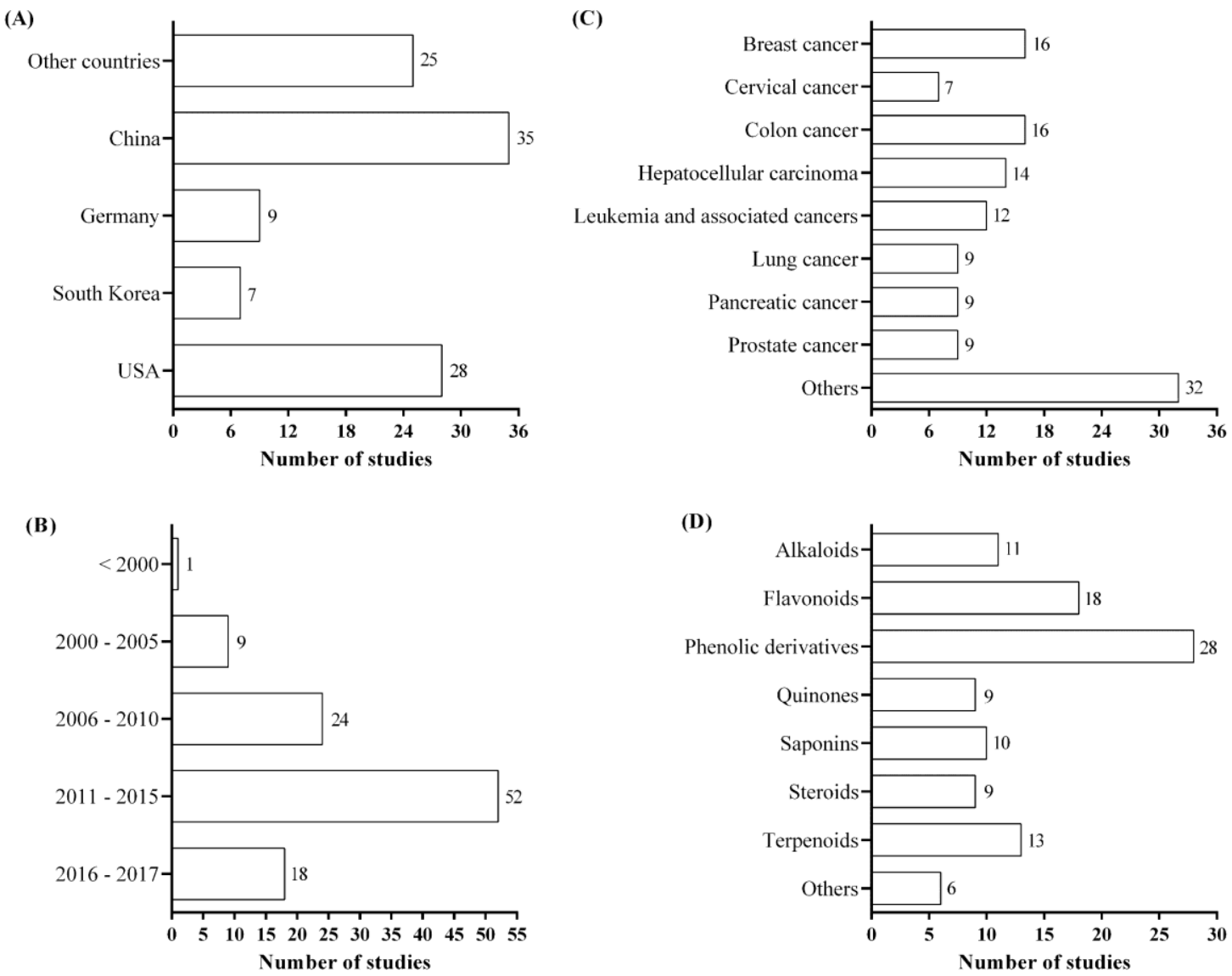

Figure 3. Distribution of the selected studies by country (A), year of publication (B), type of cancer studied (C) and chemical class of natural product evaluated as chemosensitizer (D). 
Our systematic review consisted of 67 in vitro studies, 6 in vivo studies, and 31 reports presenting in vitro and in vivo outcomes. In vitro investigations included biochemical and molecular analysis, specially colorimetric and enzymatic assays, flow cytometry, western blot and immunofluorescence techniques. In vivo reports were performed using allograft or xenograft model, as shown in Table 2. In general, natural compounds potentiated the antitumor effect of chemotherapeutics by reducing tumor volume and weight. In some cases, synergistic inhibition of metastasis and increased apoptosis index were also observed. Combinatorial treatments were performed on the same day or on alternate days for 1 to 4 weeks. The used chemotherapy drugs varied according to the type of cancer studied. All natural products tested in vivo were also assayed in vitro, providing relevant findings on molecular targets implicated in their pharmacological effect. The chemical structures of these compounds are shown in Figure 4.

Concerning to methodological quality, all in vivo studies were carefully analyzed through a standard checklist adapted for preclinical trials. As shown in Figure 5, all studies described the objectives, outcomes to be measured and main findings obtained. In general, combinatorial treatments (chemosensitizer and conventional chemotherapeutic, doses, routes of administration and frequency of treatment) were properly reported. Most of the studies (31 reports, 83.8\%) have also reported randomization of animal allocation. On the other hand, none of the included articles reported sample size calculations. In addition, no information on blinding strategy was provided. 
Table 2. In vivo studies involving natural compounds as chemosensitizer agents.

\begin{tabular}{|c|c|c|c|c|c|c|c|c|}
\hline Chemosensitizer & Dose (route) & $\begin{array}{l}\text { Combined } \\
\text { drug }\end{array}$ & Dose (route) & $\begin{array}{l}\text { Model } \\
\text { (animal/sex) }\end{array}$ & Main outcomes (Cancer) & $\overline{\mathbf{R}}$ & $\mathbf{B}$ & Reference \\
\hline $\begin{array}{l}3,3^{\prime}- \\
\text { diindolylmethane }\end{array}$ & $\begin{array}{l}5 \mathrm{mg} / \text { day } \\
\text { (p.o.) }\end{array}$ & OXP & $\begin{array}{l}15 \mathrm{mg} / \mathrm{kg} \\
\text { (i.v.) }\end{array}$ & $\begin{array}{l}\text { Xenograft } \\
(\mathrm{Mi} / \mathrm{F})\end{array}$ & $\begin{array}{l}\text { Synergistic decrease in } \\
\text { tumor weight and } \\
\text { appearance of nodal } \\
\text { metastasis (pancreatic } \\
\text { cancer) }\end{array}$ & $\mathrm{Y}$ & $\mathrm{N}$ & $\begin{array}{l}\text { Banerjee et al. } \\
(2009) \text { [29] }\end{array}$ \\
\hline \multirow[t]{2}{*}{ Cucurbitacin B } & $\begin{array}{l}0.5 \text { or } 1 \\
\mathrm{mg} / \mathrm{kg} / \mathrm{day} \\
\text { (i.p.) }\end{array}$ & GCT & $\begin{array}{l}25 \mathrm{mg} / \mathrm{kg} / \mathrm{day} \\
\text { (i.p.) }\end{array}$ & $\begin{array}{l}\text { Xenograft } \\
(\mathrm{Mi} / \mathrm{F})\end{array}$ & $\begin{array}{l}\text { Synergistic decrease in } \\
\text { tumor volume and weight, } \\
\text { synergistic inhibition of } \\
\text { metastasis (pancreatic } \\
\text { cancer) }\end{array}$ & $\mathrm{Y}$ & $\mathrm{N}$ & $\begin{array}{l}\text { Iwanski et al. } \\
\text { (2010) [112] }\end{array}$ \\
\hline & $\begin{array}{l}0.5 \text { or } 1 \\
\mathrm{mg} / \mathrm{kg} \text { (i.p.) }\end{array}$ & MET & $\begin{array}{l}50 \text { or } 150 \\
\mathrm{mg} / \mathrm{kg} \text { (i.p.) }\end{array}$ & $\begin{array}{l}\text { Xenograft } \\
(\mathrm{Mi} / \mathrm{F})\end{array}$ & $\begin{array}{l}\text { Synergistic decrease in } \\
\text { tumor volume and weight } \\
\text { (osteosarcoma) }\end{array}$ & $\mathrm{Y}$ & $\mathrm{N}$ & $\begin{array}{l}\text { Lee et al. } \\
\text { (2011) [113] }\end{array}$ \\
\hline \multirow[t]{2}{*}{ Curcumin } & $\begin{array}{l}2 \% \text { w/w/day } \\
\text { (p.o.) }\end{array}$ & PTX & $\begin{array}{l}10 \\
\mathrm{mg} / \mathrm{kg} / \text { week } \\
\text { (i.p.) }\end{array}$ & $\begin{array}{l}\text { Xenograft } \\
(\mathrm{Mi} / \mathrm{F})\end{array}$ & $\begin{array}{l}\text { Synergistic inhibition of } \\
\text { breast cancer metastasis to } \\
\text { the lung (breast cancer) }\end{array}$ & $\mathrm{Y}$ & $\mathrm{N}$ & $\begin{array}{l}\text { Aggarwal et al. } \\
\text { (2005) [67] }\end{array}$ \\
\hline & $\begin{array}{l}1 \mathrm{~g} / \mathrm{kg} / \text { day } \\
\text { (p.o.) }\end{array}$ & $\mathrm{CCT}$ & $\begin{array}{l}60 \\
\mathrm{mg} / \mathrm{kg} / \text { twice } \\
\text { weekly (p.o.) }\end{array}$ & $\begin{array}{l}\text { Xenograft } \\
(\mathrm{Mi} / \mathrm{M})\end{array}$ & $\begin{array}{l}\text { Synergistic decrease in } \\
\text { tumor volume, synergistic } \\
\text { inhibition of metastasis } \\
\text { (colon cancer) }\end{array}$ & $\mathrm{Y}$ & $\mathrm{N}$ & $\begin{array}{l}\text { Kunnumakkara } \\
\text { et al. (2009) } \\
\text { [72] }\end{array}$ \\
\hline $\begin{array}{l}\text { Curcumin (liposomal } \\
\text { formulation) }\end{array}$ & $\begin{array}{l}40 \\
\mathrm{mg} / \mathrm{kg} / \text { thrice }\end{array}$ & OXP & $\begin{array}{l}5 \\
\mathrm{mg} / \mathrm{kg} / \text { thrice }\end{array}$ & $\begin{array}{l}\text { Xenograft } \\
(\mathrm{Mi} / \mathrm{F})\end{array}$ & $\begin{array}{l}\text { Synergistic decrease in } \\
\text { tumor volume, synergistic }\end{array}$ & $\mathrm{Y}$ & $\mathrm{N}$ & $\begin{array}{l}\text { Li et al. (2007) } \\
\text { [69] }\end{array}$ \\
\hline
\end{tabular}




\begin{tabular}{|c|c|c|c|c|c|c|c|c|}
\hline & weekly (i.v.) & & weekly (i.p.) & & $\begin{array}{l}\text { inhibition of angiogenesis } \\
\text { (colon cancer) }\end{array}$ & & & \\
\hline & $\begin{array}{l}25 \\
\mathrm{mg} / \mathrm{kg} / \text { thrice } \\
\text { weekly (i.p.) }\end{array}$ & PTX & $\begin{array}{l}10 \\
\text { mg/kg/twice } \\
\text { weekly (i.p.) }\end{array}$ & $\begin{array}{l}\text { Xenograft } \\
(\mathrm{Mi} / \mathrm{F})\end{array}$ & $\begin{array}{l}\text { Synergistic decrease in } \\
\text { tumor volume, improvement } \\
\text { of apoptosis (cervical } \\
\text { cancer) }\end{array}$ & Y & $\mathrm{N}$ & $\begin{array}{l}\text { Sreekanth et } \\
\text { al. (2011) [76] }\end{array}$ \\
\hline \multirow[t]{2}{*}{$\begin{array}{l}\text { Epigallocatechin-3- } \\
\text { gallate }\end{array}$} & $\begin{array}{l}228 \\
\mathrm{mg} / \mathrm{kg} / \text { week } \\
\text { (i.p.) }\end{array}$ & $\begin{array}{l}\text { DTX + } \\
\text { PTX }\end{array}$ & $\begin{array}{l}5 \text { or } 12.5+ \\
15 \\
\mathrm{mg} / \mathrm{kg} / \text { week } \\
\text { (i.p.) }\end{array}$ & $\begin{array}{l}\text { Xenograft } \\
\text { (Mi/NR) }\end{array}$ & $\begin{array}{l}\text { Synergistic decrease in } \\
\text { tumor volume (prostate } \\
\text { cancer) }\end{array}$ & $\mathrm{Y}$ & $\mathrm{N}$ & $\begin{array}{l}\text { Stearns and } \\
\text { Wang }(2011) \\
{[51]}\end{array}$ \\
\hline & $\begin{array}{l}1.5 \mathrm{mg} / \text { day } \\
\text { (i.p.) }\end{array}$ & $\mathrm{CCT}$ & $\begin{array}{l}200 \\
\mathrm{mg} / \mathrm{kg} / \mathrm{day} \\
\text { (p.o.) }\end{array}$ & $\begin{array}{l}\text { Xenograft } \\
(\mathrm{Mi} / \mathrm{F})\end{array}$ & $\begin{array}{l}\text { Synergistic decrease in } \\
\text { tumor volume and inhibition } \\
\text { of microvessel formation } \\
\text { (gastric cancer) }\end{array}$ & Y & $\mathrm{N}$ & $\begin{array}{l}\text { Wu et al. } \\
\text { (2012) [53] }\end{array}$ \\
\hline Escin & $\begin{array}{l}2 \mathrm{mg} / \mathrm{kg} / \text { day } \\
\text { (i.p.) }\end{array}$ & GCT & $\begin{array}{l}100 \\
\text { mg/kg/twice } \\
\text { weekly (i.p.) }\end{array}$ & $\begin{array}{l}\text { Xenograft } \\
(\mathrm{Mi} / \mathrm{M})\end{array}$ & $\begin{array}{l}\text { Synergistic decrease in } \\
\text { tumor volume, improvement } \\
\text { of apoptosis (pancreatic } \\
\text { cancer) }\end{array}$ & $\mathrm{Y}$ & $\mathrm{N}$ & $\begin{array}{l}\text { Wang et al. } \\
\text { (2012b) [103] }\end{array}$ \\
\hline Genistein & $\begin{array}{l}1.3 \\
\mathrm{mg} / \mathrm{kg} / \mathrm{day} \\
\text { (i.p.) }\end{array}$ & $5 \mathrm{FU}$ & $\begin{array}{l}60 \mathrm{mg} / \mathrm{kg} / \mathrm{day} \\
\text { (i.p.) }\end{array}$ & $\begin{array}{l}\text { Xenograft } \\
(\mathrm{Mi} / \mathrm{F})\end{array}$ & $\begin{array}{l}\text { Synergistic decrease in } \\
\text { tumor volume, improvement } \\
\text { of apoptosis (pancreatic } \\
\text { cancer) }\end{array}$ & $\mathrm{Y}$ & $\mathrm{N}$ & $\begin{array}{l}\text { Suzuki et al. } \\
(2014)[55]\end{array}$ \\
\hline Ginsenoside Rg3 & $\begin{array}{l}6 \mathrm{mg} / \mathrm{kg} / \mathrm{day} \\
\text { (p.o.) }\end{array}$ & PTX + CIP & $\begin{array}{l}10+5 \\
\mathrm{mg} / \mathrm{kg} / \mathrm{day} \\
\text { (i.p.) }\end{array}$ & $\begin{array}{l}\text { Xenograft } \\
(\mathrm{Mi} / \mathrm{F})\end{array}$ & $\begin{array}{l}\text { Synergistic decrease in } \\
\text { tumor volume and weight } \\
\text { (esophageal carcinoma) }\end{array}$ & $\mathrm{Y}$ & $\mathrm{N}$ & $\begin{array}{l}\text { Chang et al. } \\
\text { (2014) [106] }\end{array}$ \\
\hline
\end{tabular}




$\begin{array}{lllll}\begin{array}{l}10 \mathrm{mg} / \mathrm{kg} / \mathrm{day} \\ \text { (p.o.) }\end{array} & \text { PTX } & \begin{array}{l}20 \mathrm{mg} / \mathrm{kg} / \mathrm{day} \\ \text { (p.o.) }\end{array} & \begin{array}{l}\text { Xenograft } \\ (\mathrm{Mi} / \mathrm{F})\end{array} & \begin{array}{l}\text { Synergistic decrease in } \\ \text { tumor volume (breast } \\ \text { cancer) }\end{array}\end{array} \begin{array}{lll}\text { Yang et al. } \\ (2012)[104]\end{array}$

$6 \mathrm{mg} / \mathrm{kg} / \mathrm{day} \quad$ PTX

$10 \quad$ Xenograft

Synergistic decrease in

Y N Yuan et al. $\mathrm{mg} / \mathrm{kg} /$ week (Mi/NR) tumor volume and weight, (2017) [109] (p.o.) (i.p.) improvement of apoptosis (breast cancer)

\begin{tabular}{|c|c|c|c|c|c|c|c|c|}
\hline Isoxanthohumol & $\begin{array}{l}20 \mathrm{mg} / \mathrm{kg} / \text { day } \\
(\mathrm{NR})\end{array}$ & PTX & $\begin{array}{l}3 \mathrm{mg} / \mathrm{kg} \\
(\mathrm{NR})\end{array}$ & $\begin{array}{l}\text { Allograft } \\
(\mathrm{Mi} / \mathrm{NR})\end{array}$ & $\begin{array}{l}\text { Synergistic decrease in } \\
\text { tumor volume (melanoma) }\end{array}$ & $\mathrm{Y}$ & $\mathrm{N}$ & $\begin{array}{l}\text { Krajnovic et } \\
\text { al. (2016) [60] }\end{array}$ \\
\hline \multirow[t]{2}{*}{ Lupeol } & $\begin{array}{l}20 \\
\mathrm{mg} / \mathrm{kg} / \text { thrice } \\
\text { weekly (i.p.) }\end{array}$ & S14161 & $\begin{array}{l}20 \mathrm{mg} / \mathrm{kg} / \text { day } \\
\text { (i.p.) }\end{array}$ & $\begin{array}{l}\text { Xenograft } \\
(\mathrm{Mi} / \mathrm{F})\end{array}$ & $\begin{array}{l}\text { Synergistic decrease in } \\
\text { tumor volume } \\
\text { (hepatocellular carcinoma) }\end{array}$ & $\mathrm{Y}$ & $\mathrm{N}$ & $\begin{array}{l}\text { Liu et al. } \\
(2013)[128]\end{array}$ \\
\hline & $\begin{array}{l}30 \mathrm{mg} / \mathrm{kg} / \text { day } \\
\text { (i.p.) }\end{array}$ & $5 \mathrm{FU}$ & $\begin{array}{l}10 \mathrm{mg} / \mathrm{kg} / \text { day } \\
\text { (i.p.) }\end{array}$ & $\begin{array}{l}\text { Xenograft } \\
(\mathrm{Mi} / \mathrm{NR})\end{array}$ & $\begin{array}{l}\text { Synergistic decrease in } \\
\text { tumor volume and weight, } \\
\text { improvement of apoptosis } \\
\text { (gastric cancer) }\end{array}$ & $\mathrm{N}$ & $\mathrm{N}$ & $\begin{array}{l}\text { Liu et al. } \\
(2016)[130]\end{array}$ \\
\hline Myricetin & $25 \mathrm{mg} / \mathrm{kg}$ & $5 \mathrm{FU}$ & $20 \mathrm{mg} / \mathrm{kg}$ & $\begin{array}{l}\text { Xenograft } \\
(\mathrm{Mi} / \mathrm{NR})\end{array}$ & $\begin{array}{l}\text { Synergistic decrease in } \\
\text { tumor volume (esophageal } \\
\text { carcinoma) }\end{array}$ & $\mathrm{N}$ & $\mathrm{N}$ & $\begin{array}{l}\text { Wang et al. } \\
(2014 a)[56]\end{array}$ \\
\hline Naringenin & $\begin{array}{l}50 \mathrm{mg} / \mathrm{kg} / \mathrm{day} \\
\text { (p.o.) }\end{array}$ & DOX & $\begin{array}{l}5 \\
\mathrm{mg} / \mathrm{kg} / \text { week } \\
\text { (p.o.) }\end{array}$ & $\begin{array}{l}\text { Allograft } \\
(\mathrm{Mi} / \mathrm{F})\end{array}$ & $\begin{array}{l}\text { Synergistic decrease in } \\
\text { tumor volume (lung cancer) }\end{array}$ & $\mathrm{N}$ & $\mathrm{N}$ & $\begin{array}{l}\text { Zhang et al. } \\
\text { (2009) [49] }\end{array}$ \\
\hline Noscapine & 300 & GCT & $30 \mathrm{mg} / \mathrm{kg}$ & Xenograft & Synergistic decrease in & $\mathrm{Y}$ & $\mathrm{N}$ & Chougule et al. \\
\hline
\end{tabular}




\begin{tabular}{|c|c|c|c|c|c|c|c|c|}
\hline & $\begin{array}{l}\mathrm{mg} / \mathrm{kg} / \mathrm{day} \\
\text { (p.o.) }\end{array}$ & & (i.v.) & $(\mathrm{Mi} / \mathrm{F})$ & $\begin{array}{l}\text { tumor volume and inhibition } \\
\text { of angiogenesis in tumor } \\
\text { tissue (lung cancer) }\end{array}$ & & & (2011) [31] \\
\hline & $\begin{array}{l}200 \\
\mathrm{mg} / \mathrm{kg} / \text { day } \\
\text { (i.g.) }\end{array}$ & TMZ or CIP & $\begin{array}{l}2 \mathrm{mg} / \mathrm{kg} / \mathrm{day} \\
\text { (i.p.) }\end{array}$ & $\begin{array}{l}\text { Xenograft } \\
(\mathrm{Mi} / \mathrm{F})\end{array}$ & $\begin{array}{l}\text { Synergistic decrease in } \\
\text { tumor volume and weight } \\
\text { (glioblastoma) }\end{array}$ & $\mathrm{Y}$ & $\mathrm{N}$ & $\begin{array}{l}\text { Qi et al. (2013) } \\
\text { [33] }\end{array}$ \\
\hline \multirow[t]{2}{*}{ Oxymatrine } & $\begin{array}{l}40 \mathrm{mg} / \mathrm{kg} / \mathrm{day} \\
\text { (i.p.) }\end{array}$ & $5 \mathrm{FU}$ & $\begin{array}{l}10 \mathrm{mg} / \mathrm{kg} / \mathrm{day} \\
\text { (i.p.) }\end{array}$ & $\begin{array}{l}\text { Xenograft } \\
\text { (Mi/NR) }\end{array}$ & $\begin{array}{l}\text { Synergistic decrease in } \\
\text { tumor volume and weight } \\
\text { (hepatocellular carcinoma) }\end{array}$ & $\mathrm{N}$ & $\mathrm{N}$ & $\begin{array}{l}\text { Liu et al. } \\
(2015 a)[36]\end{array}$ \\
\hline & $\begin{array}{l}1,2 \text { or } 4 \\
\text { g/l/thrice } \\
\text { weekly (i.p.) }\end{array}$ & NM-3 & $\begin{array}{l}10 \\
\text { mg/kg/thrice } \\
\text { weekly (i.p.) }\end{array}$ & $\begin{array}{l}\text { Xenograft } \\
\text { (Mi/NR) }\end{array}$ & $\begin{array}{l}\text { Synergistic decrease in } \\
\text { tumor volume (gastric } \\
\text { cancer) }\end{array}$ & $\mathrm{Y}$ & $\mathrm{N}$ & $\begin{array}{l}\text { Song et al. } \\
\text { (2007) [28] }\end{array}$ \\
\hline \multirow[t]{2}{*}{ Resveratrol } & $\begin{array}{l}40 \mathrm{mg} / \mathrm{kg} / \text { day } \\
\text { (p.o.) }\end{array}$ & GCT & $\begin{array}{l}25 \\
\mathrm{mg} / \mathrm{kg} / \text { twice } \\
\text { weekly (i.p.) }\end{array}$ & $\begin{array}{l}\text { Xenograft } \\
(\mathrm{Mi} / \mathrm{M})\end{array}$ & $\begin{array}{l}\text { Synergistic decrease in } \\
\text { tumor volume (pancreatic } \\
\text { cancer) }\end{array}$ & $\mathrm{Y}$ & $\mathrm{N}$ & $\begin{array}{l}\text { Harikumar et } \\
\text { al. (2009) [71] }\end{array}$ \\
\hline & $\begin{array}{l}5,10 \text { and } 15 \\
\mathrm{mg} / \mathrm{kg} / \text { day }\end{array}$ & $5 \mathrm{FU}$ & $\begin{array}{l}5,10 \text { and } 20 \\
\mathrm{mg} / \mathrm{kg}\end{array}$ & $\begin{array}{l}\text { Allograft } \\
(\mathrm{Mi} / \mathrm{NR})\end{array}$ & $\begin{array}{l}\text { Synergistic decrease in } \\
\text { tumor area (hepatocellular } \\
\text { carcinoma) }\end{array}$ & $\mathrm{Y}$ & $\mathrm{N}$ & $\begin{array}{l}\text { Wu et al. } \\
\text { (2004) [66] }\end{array}$ \\
\hline \multirow[t]{2}{*}{ Shikonin } & $\begin{array}{l}4 \mathrm{mg} / \mathrm{kg} / \mathrm{day} \\
\text { (i.p.) }\end{array}$ & CIP & $\begin{array}{l}10 \mathrm{mg} / \mathrm{kg} / \mathrm{day} \\
\text { (i.p.) }\end{array}$ & $\begin{array}{l}\text { Xenograft } \\
(\mathrm{Mi} / \mathrm{F})\end{array}$ & $\begin{array}{l}\text { Synergistic decrease in } \\
\text { tumor volume and weight } \\
\text { (colon cancer) }\end{array}$ & $\mathrm{N}$ & $\mathrm{N}$ & $\begin{array}{l}\text { He et al. } \\
\text { (2016) [97] }\end{array}$ \\
\hline & $\begin{array}{l}3 \mathrm{mg} / \mathrm{kg} / \mathrm{day} \\
\text { (i.p.) }\end{array}$ & ATO & $\begin{array}{l}10 \mathrm{mg} / \mathrm{kg} / \mathrm{day} \\
\text { (i.p.) }\end{array}$ & $\begin{array}{l}\text { Xenograft } \\
\text { (Mi/NR) }\end{array}$ & $\begin{array}{l}\text { Synergistic decrease in } \\
\text { tumor volume and weight } \\
\text { (hepatocellular carcinoma) }\end{array}$ & $\mathrm{Y}$ & $\mathrm{N}$ & $\begin{array}{l}\text { Song et al. } \\
\text { (2016) [98] }\end{array}$ \\
\hline
\end{tabular}




$\begin{array}{llll}2 \mathrm{mg} / \mathrm{kg} / \text { day } & \text { GCT } & 100 & \text { Xenograft } \\ \text { (i.p.) } & & \begin{array}{l}\text { mg/kg/twice } \\ \text { weekly (i.p.) }\end{array} & \text { (Mi/M) }\end{array}$

Synergistic decrease in

tumor volume, synergistic

inhibition of microvessel

formation and induction of apoptosis (pancreatic

cancer)

\begin{tabular}{|c|c|c|c|c|c|c|c|c|}
\hline & & & & & $\begin{array}{l}\text { formation and induction of } \\
\text { apoptosis (pancreatic } \\
\text { cancer) }\end{array}$ & & & \\
\hline Sinomenine & $\begin{array}{l}25 \\
\mathrm{mg} / \mathrm{kg} / \text { twice } \\
\text { weekly (i.t.) }\end{array}$ & $5 \mathrm{FU}$ & $\begin{array}{l}12 \\
\mathrm{mg} / \mathrm{kg} / \text { twice } \\
\text { weekly (i.t.) }\end{array}$ & $\begin{array}{l}\text { Xenograft } \\
(\mathrm{Mi} / \mathrm{M})\end{array}$ & $\begin{array}{l}\text { Synergistic decrease in } \\
\text { tumor volume and weight } \\
\text { (esophageal carcinoma) }\end{array}$ & $\mathrm{Y}$ & $\mathrm{N}$ & $\begin{array}{l}\text { Wang et al. } \\
\text { (2013a) [34] }\end{array}$ \\
\hline Steroidal saponin & $\begin{array}{l}5,10 \text { or } 15 \\
\mathrm{mg} / \mathrm{kg} / \text { day } \\
\text { (i.v.) }\end{array}$ & DOX & $\begin{array}{l}8 \mathrm{mg} / \mathrm{kg} / \mathrm{day} \\
\text { (i.v.) }\end{array}$ & $\begin{array}{l}\text { Xenograft } \\
\text { (Mi/NR) }\end{array}$ & $\begin{array}{l}\text { Synergistic decrease in } \\
\text { tumor volume } \\
\text { (hepatocellular carcinoma) }\end{array}$ & $\mathrm{N}$ & $\mathrm{N}$ & $\begin{array}{l}\text { Wang et al. } \\
\text { (2013b) [105] }\end{array}$ \\
\hline Thymoquinone & $\begin{array}{l}5 \text { or } 20 \\
\mathrm{mg} / \mathrm{kg} / \text { day } \\
\text { (s.c.) }\end{array}$ & CIP & $\begin{array}{l}2.5 \\
\mathrm{mg} / \mathrm{kg} / \text { week } \\
\text { (i.p.) }\end{array}$ & $\begin{array}{l}\text { Xenograft } \\
(\mathrm{Mi} / \mathrm{F})\end{array}$ & $\begin{array}{l}\text { Synergistic decrease in } \\
\text { tumor volume (lung cancer) }\end{array}$ & $\mathrm{Y}$ & $\mathrm{N}$ & $\begin{array}{l}\text { Jafri et al. } \\
\text { (2010) [91] }\end{array}$ \\
\hline Ursolic acid & $\begin{array}{l}250 \\
\mathrm{mg} / \mathrm{kg} / \text { day } \\
\text { (p.o.) }\end{array}$ & $\mathrm{CCT}$ & $\begin{array}{l}60 \\
\mathrm{mg} / \mathrm{kg} / \mathrm{twice} \\
\text { weekly (p.o.) }\end{array}$ & $\begin{array}{l}\text { Xenograft } \\
(\mathrm{Mi} / \mathrm{M})\end{array}$ & $\begin{array}{l}\text { Synergistic decrease in } \\
\text { tumor volume and weight, } \\
\text { synergistic inhibition of } \\
\text { metastasis (colon cancer) }\end{array}$ & $\mathrm{Y}$ & $\mathrm{N}$ & $\begin{array}{l}\text { Prasad et al. } \\
(2012)[124]\end{array}$ \\
\hline Withaferin A & $\begin{array}{l}2 \mathrm{mg} / \mathrm{kg} / \mathrm{day} \\
\text { (i.p.) }\end{array}$ & DOX & $\begin{array}{l}1 \mathrm{mg} / \mathrm{kg} / \text { day } \\
\text { (i.p.) }\end{array}$ & $\begin{array}{l}\text { Xenograft } \\
\text { (Mi/NR) }\end{array}$ & $\begin{array}{l}\text { Synergistic decrease in } \\
\text { tumor volume and weight, } \\
\text { synergistic inhibition of } \\
\text { microvessel formation and } \\
\text { induction of autophagy } \\
\text { (ovarian cancer) }\end{array}$ & $\mathrm{Y}$ & $\mathrm{N}$ & $\begin{array}{l}\text { Fong et al. } \\
\text { (2012) [115] }\end{array}$ \\
\hline
\end{tabular}

Y N Wang et al.

(2014b) [95] 


\begin{tabular}{|c|c|c|c|c|c|c|c|c|}
\hline & $\begin{array}{l}3 \mathrm{mg} / \mathrm{kg} / \text { day } \\
\text { (i.p.) }\end{array}$ & OXP & $\begin{array}{l}10 \\
\text { mg/kg/twice } \\
\text { weekly (i.p.) }\end{array}$ & $\begin{array}{l}\text { Xenograft } \\
(\mathrm{Mi} / \mathrm{M})\end{array}$ & $\begin{array}{l}\text { Synergistic decrease in } \\
\text { tumor volume and weight, } \\
\text { synergistic induction of } \\
\text { apoptosis (pancreatic } \\
\text { cancer) }\end{array}$ & & $\mathrm{N}$ & $\begin{array}{l}\text { Li et al. (2015) } \\
{[117]}\end{array}$ \\
\hline$\alpha$-carotene & $\begin{array}{l}5 \mathrm{mg} / \mathrm{kg} / \text { day } \\
\text { (p.o.) }\end{array}$ & PTX & $\begin{array}{l}6 \mathrm{mg} / \mathrm{kg} / \mathrm{day} \\
\text { (i.p.) }\end{array}$ & $\begin{array}{l}\text { Xenograft } \\
(\mathrm{Mi} / \mathrm{M})\end{array}$ & $\begin{array}{l}\text { Synergistic decrease in lung } \\
\text { metastasis (lung cancer) }\end{array}$ & $\mathrm{Y}$ & $\mathrm{N}$ & $\begin{array}{l}\text { Liu et al. } \\
(2015 b)[40]\end{array}$ \\
\hline$\beta$-carotene & $\begin{array}{l}5 \\
\mathrm{mg} / \mathrm{kg} / \text { thrice } \\
\text { weekly (i.g.) }\end{array}$ & $5 \mathrm{FU}$ & $\begin{array}{l}5 \\
\mathrm{mg} / \mathrm{kg} / \text { thrice } \\
\text { weekly (i.p.) }\end{array}$ & $\begin{array}{l}\text { Xenograft } \\
(\mathrm{Mi} / \mathrm{M})\end{array}$ & $\begin{array}{l}\text { Synergistic decrease in } \\
\text { tumor volume and weight, } \\
\text { improvement of apoptosis } \\
\text { (esophageal carcinoma) }\end{array}$ & $\mathrm{Y}$ & $\mathrm{N}$ & $\begin{array}{l}\text { Zhang et al. } \\
\text { (2016) [41] }\end{array}$ \\
\hline $\begin{array}{l}\Delta^{9}- \\
\text { Tetrahydrocannabin } \\
\text { ol }\end{array}$ & $\begin{array}{l}15 \mathrm{mg} / \mathrm{kg} \\
\text { (i.t.) }\end{array}$ & TMZ & $5 \mathrm{mg} / \mathrm{kg}$ (i.t.) & $\begin{array}{l}\text { Xenograft } \\
(\mathrm{Mi} / \mathrm{NR})\end{array}$ & $\begin{array}{l}\text { Synergistic decrease in } \\
\text { tumor volume and weight } \\
\text { (glioma) }\end{array}$ & $\mathrm{Y}$ & $\mathrm{N}$ & $\begin{array}{l}\text { Torres et al. } \\
(2011) \text { [123] }\end{array}$ \\
\hline
\end{tabular}




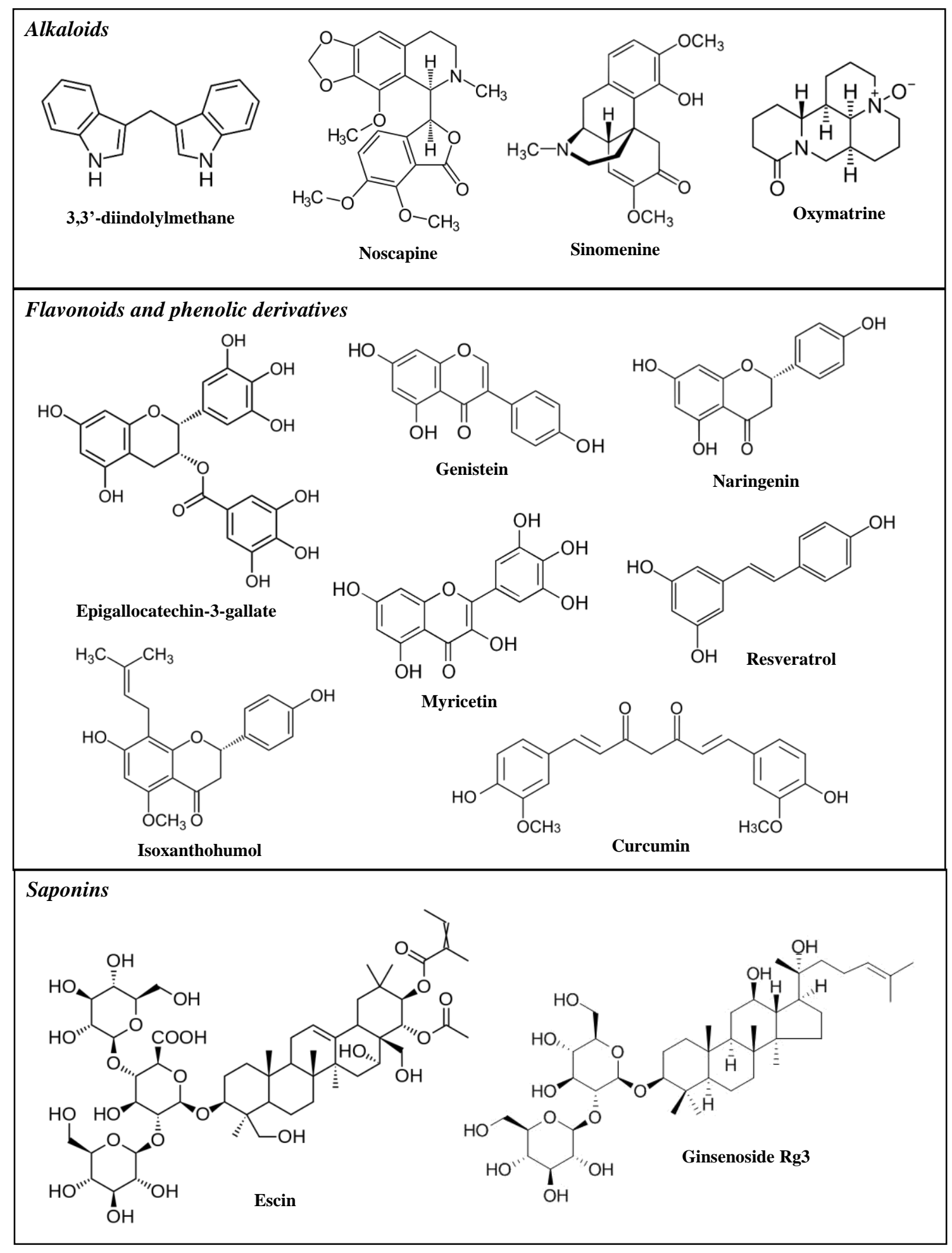

(continued on next page) 
(continued)

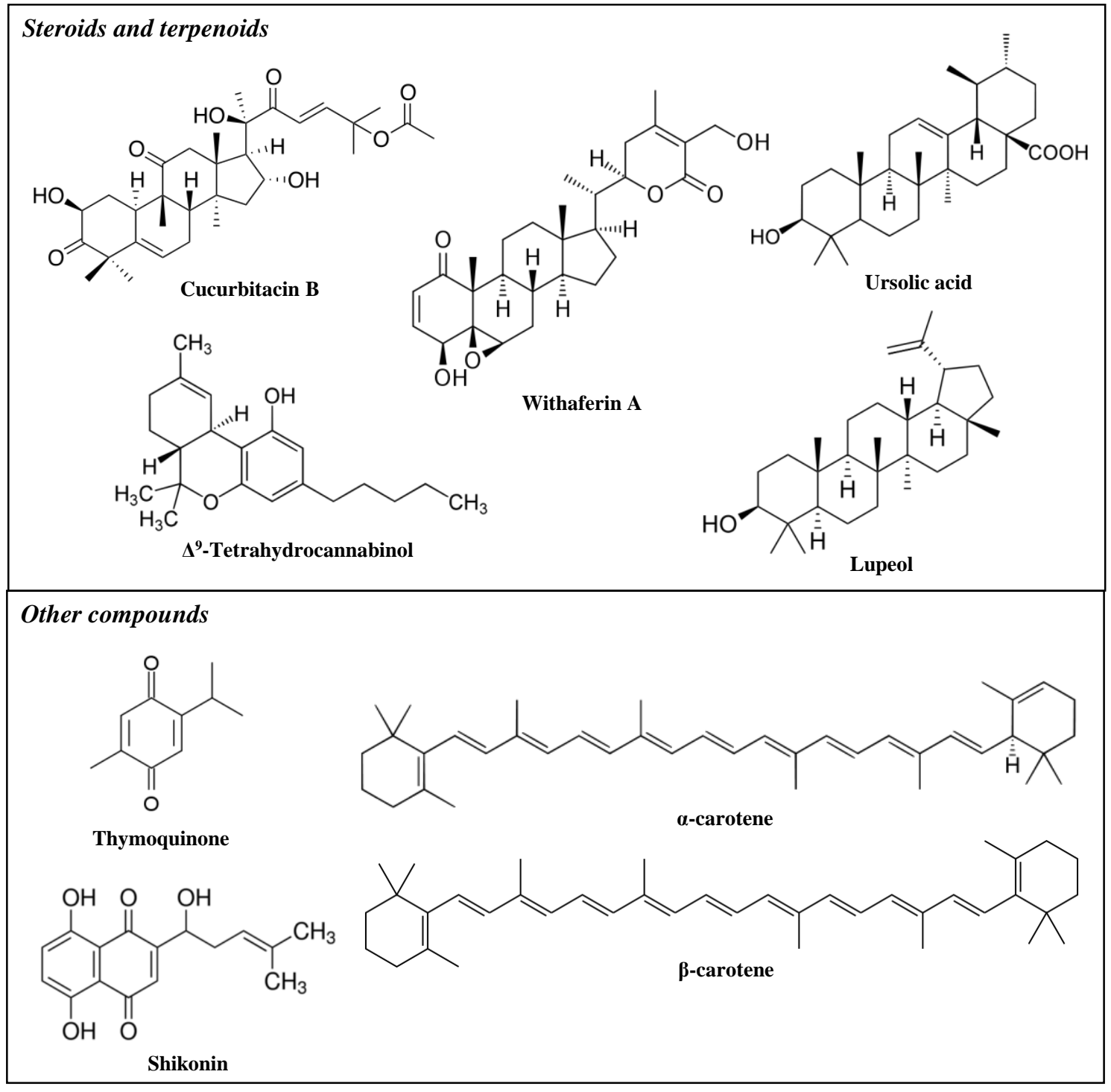

Figure 4. Chemical structure of the major natural compounds evaluated as chemosensitizer agents (in vitro and in vivo evidences). 


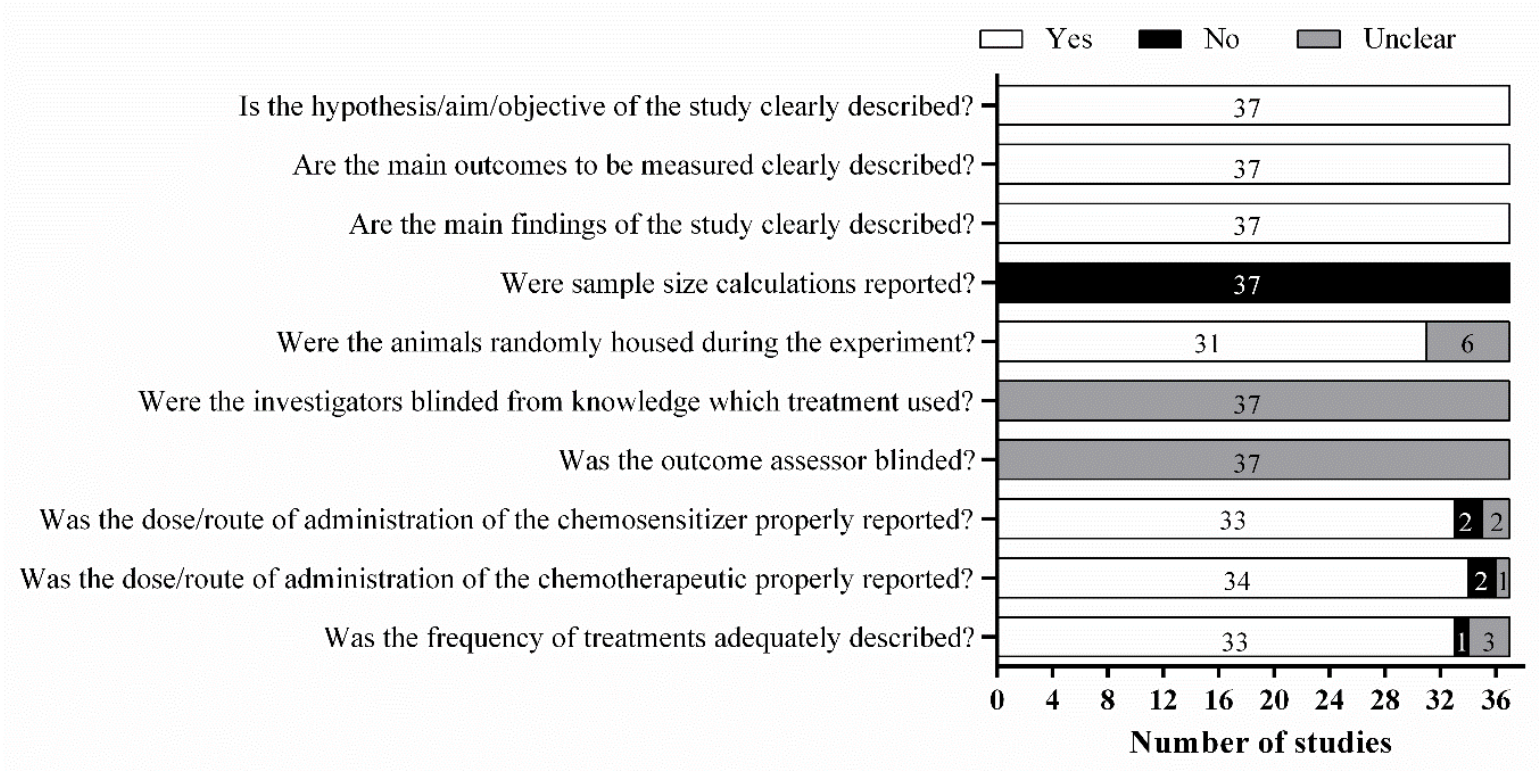

Figure 5. Methodological quality assessment of included in vivo studies. Light bars indicate the proportion of articles that met each criterion; dark bars indicate the proportion of studies that did not and white gray bars indicate the proportion of studies with unclear or insufficient answers.

\section{Discussion}

Cancer therapy is based on the use of one or more treatment strategies, including surgical removal of the tumor, radiotherapy, immunotherapy, phototherapy and chemotherapy. Although chemotherapy is recognized as one of the most effective strategies in the treatment of various types of cancer, the phenomenon of chemoresistance has become increasingly frequent, representing an obstacle to the use of anticancer drugs [132]. Tumor cells may develop a multidrug-resistant phenotype depending on the carcinogenic process per se, or even due to exposure to conventional chemotherapeutics [133]. In this sense, chemosensitization represents an alternative for overcoming chemoresistance. It consists in the use of molecules capable of improving the activity of another through the modulation of one or more mechanisms of resistance (Figure 1).

Historically, natural products have been shown to be more effective than conventional anticancer drugs because of their multi-target potential and low toxicity. Such compounds are already widely known as promising anti-tumor and chemopreventive agents. Fortunately, several research groups have also investigated the 
role of natural products in sensitizing tumor cells. In this systematic review, most of the included studies were published after 2011 (Figure 3), indicating that the use of natural compounds as chemosensitizer agents is still recent.

Interestingly, China has been the country that most explores the use of natural products as chemosensitizers (Figure 3). In fact, Traditional Chinese Medicine (TCM) has contributed to the development of new pharmaceutical products based on plant extracts or even molecules with unique chemical structures and innovative mechanisms of action [134]. In cancer therapy, TCM has provided molecules with antitumor and chemopreventive properties [135] and, more recently, chemosensitizing potential. Shikonin, a natural naphthoquinone derived from the Chinese medicinal herb Lithospermum erythrorhizon, showed synergistic effect with gemcitabine, cisplatin and arsenic trioxide against pancreatic [95], colon [97] and hepatocellular [98] cancer, respectively. Song et al. [28] and Liu et al. [36] have also demonstrated the chemosensitizing effect of oxymatrine, one of the major components extracted from Sophora flavescens, widely used in TCM. In addition, several phenolic derivatives and flavonoids commonly found in Chinese medicinal plants were investigated as chemosensitizers, including resveratrol [65], curcumin [64], naringenin [49] and myricetin [56].

Concerning to in vivo studies included, natural products were investigated using xenograft model. In this model, human tumor cells are transplanted via subcutaneous inoculation or into the organ type in which the tumor originated, into immunocompromised animals that do not reject human cells [136]. Xenograft models have been used not only to determine the in vivo activity of new anticancer drugs, but also to determine drug dose, treatment schedules and routes of administration [137]. In this context, in vivo reports included in this review were appropriately described. In addition to in vitro protocols, these models offer a wealth of information on the mechanisms of action involved in the chemosensitizing effect of natural products.

However, animal experiments should be well designed, efficiently executed and data must to be correctly analyzed and interpreted [138]. Regarding the methodological quality assessment, we found that most of studies were conducted randomly, but no information on blinding was provided (Figure 5). In addition, no study reported sample size calculations. Although these parameters are often required in clinical trials, the need of randomization and blinding have been strongly recommended for preclinical protocols in order to minimize the risk of bias and avoid unexpected outcomes in 
clinical trials [139-141]. For this reason, we consider that the in vivo studies included in this review presented moderate methodological quality.

In general, phenolic derivatives and flavonoids were the most cited compounds (Figure 3). Curcumin, resveratrol and epigallocatechin-3-gallate have been extensively evaluated in combinatorial treatment with clinically used chemotherapeutics. These compounds are widely found in various medicinal plants and foods, such as red wine, fruits, vegetables and spices. The use of these molecules has been increasingly encouraged in cancer treatment mainly because of their low toxicity and immediate availability. Besides, phenolic compounds possess a strong antitumor activity by modulating different pathways involved in cell proliferation, invasion, metastasis and angiogenesis [19-22]. Usually, when cancer cells were treated by natural products in combination with chemotherapeutic drugs, there was an additive cytotoxic effect caused by the activation of alternative signaling pathways that induce cell death, or even by increasing the residence time of the anticancer drug in the cell, improving its performance.

Next, we selected the natural compounds most cited in this review in order to better understand the different mechanisms of action involved in the sensitization of tumor cells. All findings described below were extracted from in vitro and in vivo included studies.

\subsection{Curcumin}

Curcumin (diferuloyl methane) is a naturally occurring phenolic pigment found in rhizomes of Curcuma longa Linn., commonly known as turmeric. Usually, curcumin content in turmeric varies from 1 to $5 \%$ and it is widely used in foods, as a cosmetic ingredient, and in some medicinal preparations [142]. It has potent anti-inflammatory, anticancer and chemopreventive properties, but without exhibiting toxic effects in animal models even at high doses [143-145]. Curcumin has demonstrated multiple anticancer effects, including inhibition of cell proliferation, induction of apoptosis, inhibition of angiogenesis and metastasis. Several mechanisms have been implicated in these effects, such as activation of pro-apoptotic proteins and inhibition of nuclear factor $\kappa \mathrm{B}(\mathrm{NF}-\kappa \mathrm{B})$ and phosphatidylinositol (PI)3-kinase/Akt (PI3K/Akt) pathways, commonly activated in multiresistant tumor cells [20]. In figure 6, we show the main mechanisms involved in the chemosensitizing effect of curcumin. 
In contrast to healthy cells, NF- $\mathrm{B}$ pathway is constitutively active in the majority of solid and hematopoietic tumor cell lines. Additionally, chemotherapeutic agents and pro-inflammatory cytokines also activate NF- $\kappa \mathrm{B}$ over time, contributing to chemoresistance of tumor cells. NF- $\mathrm{BB}$ is a tumorigenic transcription factor associated with evasion of apoptosis, sustained cell proliferation, invasion, metastasis and angiogenesis. It is a complex protein composed of different subunits (p50, p52, p65, RelB and c-Rel), mainly p50/p65. Under normal conditions, NF- $\kappa B$ is retained in the cytoplasm by its interaction with inhibitors of $\kappa \mathrm{B}(\mathrm{I} \kappa \mathrm{B} \alpha, \mathrm{I} \kappa \mathrm{B} \beta$ or $\mathrm{I} \kappa \mathrm{B} \varepsilon)$. However, I $\mathrm{K} B$ kinases (IKKs) are able to phosphorylate I $\mathrm{KB}$ portion, resulting in its subsequent ubiquitination and proteasome-mediated degradation, and consequently in the release of $\mathrm{NF}-\kappa \mathrm{B}$, which then translocates to the nucleus [74, 146]. In this review, we have identified that curcumin down-regulates NF- $\mathrm{BB}$ activation induced by chemotherapeutic agents, such as paclitaxel [67,68,75], 5-fluorouracil [82] and capecitabine [72] in cervical, breast and colon cancer. Western blot and immunohistochemical analysis

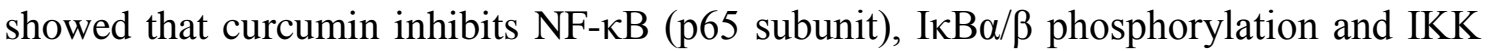
activation, resulting in synergistic antitumor effect when combined with conventional chemotherapeutic agents [64,87].

$\mathrm{NF}-\kappa \mathrm{B}$ can also be stimulated via the PI3K/Akt signaling pathway. Initially, exposure to cellular survival factors (growth factors, cytokines, etc.) hyperactivates PI3K, leading to high Akt activation, conferring cell survival and resistance to chemotherapy-induced apoptosis. In fact, Akt protects apoptosis by stimulating antiapoptotic proteins (e.g. survinin) and inhibiting pro-apoptotic signals (e.g. BAD). Furthermore, Akt induces the release of NF- $\kappa B$ through activation of IKK $[79,147]$. Once available, NF- $\mathrm{BB}$ upregulates the expression of multiple MDR genes in tumor cells that play a role in apoptosis, cell proliferation, invasion, metastasis and angiogenesis [72]. In this sense, pharmacological investigations have demonstrated that curcumin potentiates anticancer effects of chemotherapeutics not only by inhibiting PI3K, Akt and NF- $\kappa \mathrm{B}$ factors $[68,75,79,82]$, but also the proteins expressed by the activation of these signaling pathways, including those involved in cell proliferation (e.g. Cyclin D1, COX-2, c-Myc), invasion (e.g. MMP-9), metastasis (e.g. CXCR4 and ICAM-1) and angiogenesis (e.g. VEGF) [72,79,87]. Finally, curcumin also acts synergistically with chemotherapeutics in the induction of apoptosis through stimulation of pro-apoptotic (e.g. BAD, BID, BIM, BAX, caspases 3, 8 and 9) proteins and inhibition of anti-apoptotic proteins (e.g. Bcl-2, Bcl-xL and survinin) $[72,75,84]$. 
MDRs may also involve efflux pumps that reduce the residence time of chemotherapeutic drugs in cancer cells. Anuchapreeda et al. [63] have investigated the role of curcumin in P-glycoprotein (Pgp) expression. Pgp, also known as multidrug resistance protein, is an important transmembrane protein that pumps many foreign drugs out of cells. Many synthetic Pgp modulators successfully reverse the MDR phenotype in vitro. On the other hand, the use of these compounds has been discouraged due to their toxicity profile observed in animal models and clinical trials. In this review, Pgp expression was found to be decreased by 11, 31, 60 and $64 \%$ in response to 1, 2, 3 and 4 days of treatment with $25 \mu \mathrm{M}$ curcumin, respectively, enhancing sensitivity of KB-V1 cells (cervical cancer) to vinblastine [63]. Since curcumin is considered a safe natural product, it has served as a prototype for obtaining new Pgp-modulating drugs [89]. 


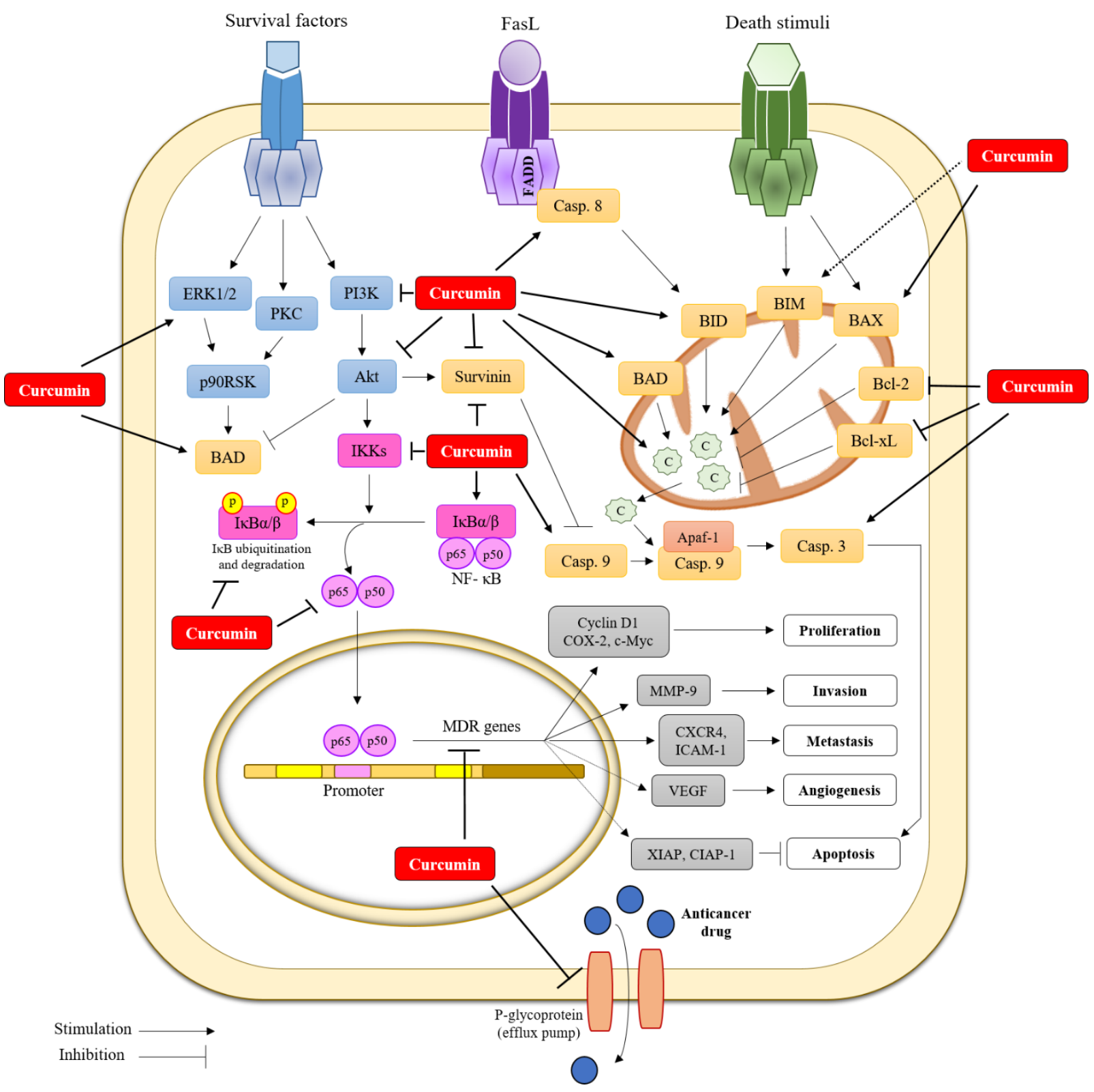

Figure 6. Molecular mechanisms of curcumin-mediated chemosensitization. Curcumin modulates signaling pathways involved in apoptosis, cell proliferation, invasion, metastasis and angiogenesis

\subsection{Resveratrol}

Resveratrol (trans-3,5,4'-trihydroxystilben) is a natural compound produced by the action of stilbene synthase in response to environmental stress, widely found in grapes, red wine, medicinal plants, various berries and nuts. This phenolic derivative possesses a wide spectrum of pharmacological activities, including anticancer properties. Resveratrol has presented an ability to target multiple signaling pathways 
implicated in tumor cell survival, inflammation, invasion, metastasis and angiogenesis [148-149]. Several studies showed its antitumor activity in human cancer from different origin, including skin, breast, prostate, liver, pancreas, colon, lung and stomach [80, 150]. In recent years, resveratrol has been evaluated not only as a chemopreventive or chemotherapeutic agent, but also as a chemosensitizer. In our review, in vitro and in vivo evidences demonstrated that resveratrol potentiates the antitumor effect of several chemotherapeutics, mainly doxorubicin [65,77,81], 5-Fluorouracil [65,66,85], etoposide [80] and gemcitabine [71].

Similar to curcumin, resveratrol sensitizes tumor cells by inhibiting the NF- $\mathrm{BB}$ signaling pathway [85], as shown in figure 7. In addition, this phenolic derivative modulates the expression of MDR genes by down-regulating targets related to cell proliferation (e.g. cyclin D1, COX-2 and c-Myc), invasion (e.g. MMP-9), metastasis (e.g. CXCR4 and ICAM-1) and angiogenesis (e.g. VEGF) [71,85]. Resveratrol also enhances the cytotoxicity of chemotherapeutics through the induction of apoptosis by regulating the expression of pro (e.g. p53, caspases 3 and 8) and anti-apoptotic (e.g. Bcl-2, Bcl-xL, XIAP and survinin) mediators in tumor cells $[65,71,80,85]$.

Díaz-Chávez et al. [81] showed that resveratrol sensitizes breast cancer cells to doxorubicin therapy by inhibiting HSP27 expression. HSP27 is present in several cell types, located mainly in the cytosol, but also in the perinuclear region, endoplasmic reticulum and nucleus. It is usually overexpressed during different stages of cell development and differentiation. High HSP27 expression has been observed in several types of cancer, suggesting that it plays an important role in cell proliferation, metastasis and chemoresistance. HSP27 acts as an independent ATP chaperone by inhibiting protein aggregation and stabilizing partially denatured proteins. In apoptosis, it interacts with mitochondrial membranes, interfering with the activation of the cytochrome-c/Apaf-1 complex and consequently preventing the activation of procaspase 9 [151].

Interestingly, resveratrol also enhances the efficacy of chemotherapeutics not only by interfering with intracellular signaling pathways, but also by modulating the expression of transmembrane proteins involved in cell proliferation and cytoskeleton stabilization. Buhrmann et al. [85] showed that resveratrol induces chemosensitization of colon cancer cells to 5-fluorouracil through up-regulation of intercellular junctions, epithelial-to-mesenchymal transition and apoptosis. In this investigation, resveratrol increased the expression of adhesion molecules, such as E-cadherin and claudin-2 (also 
involved in tight junctions), ensuring greater cell adhesion and consequently preventing mechanisms of cell proliferation. Furthermore, resveratrol significantly attenuated drug resistance by inhibiting epithelial-mesenchymal transition factors, such as vimentin. This protein is attached to the nucleus, endoplasmic reticulum and mitochondria, laterally or terminally. The vimentin filaments are associated with the nuclear and plasma membranes, maintaining the position of the nucleus and the mitotic spindle and guaranteeing flexibility to the cell. It is a component of the cytoskeleton that interacts closely with microtubules, ensuring their stabilization [152]. Once inhibited by resveratrol, vimentin disperses in aggregates, causing loss of cytoplasmic integrity and changes in cellular morphology (Figure 7). 


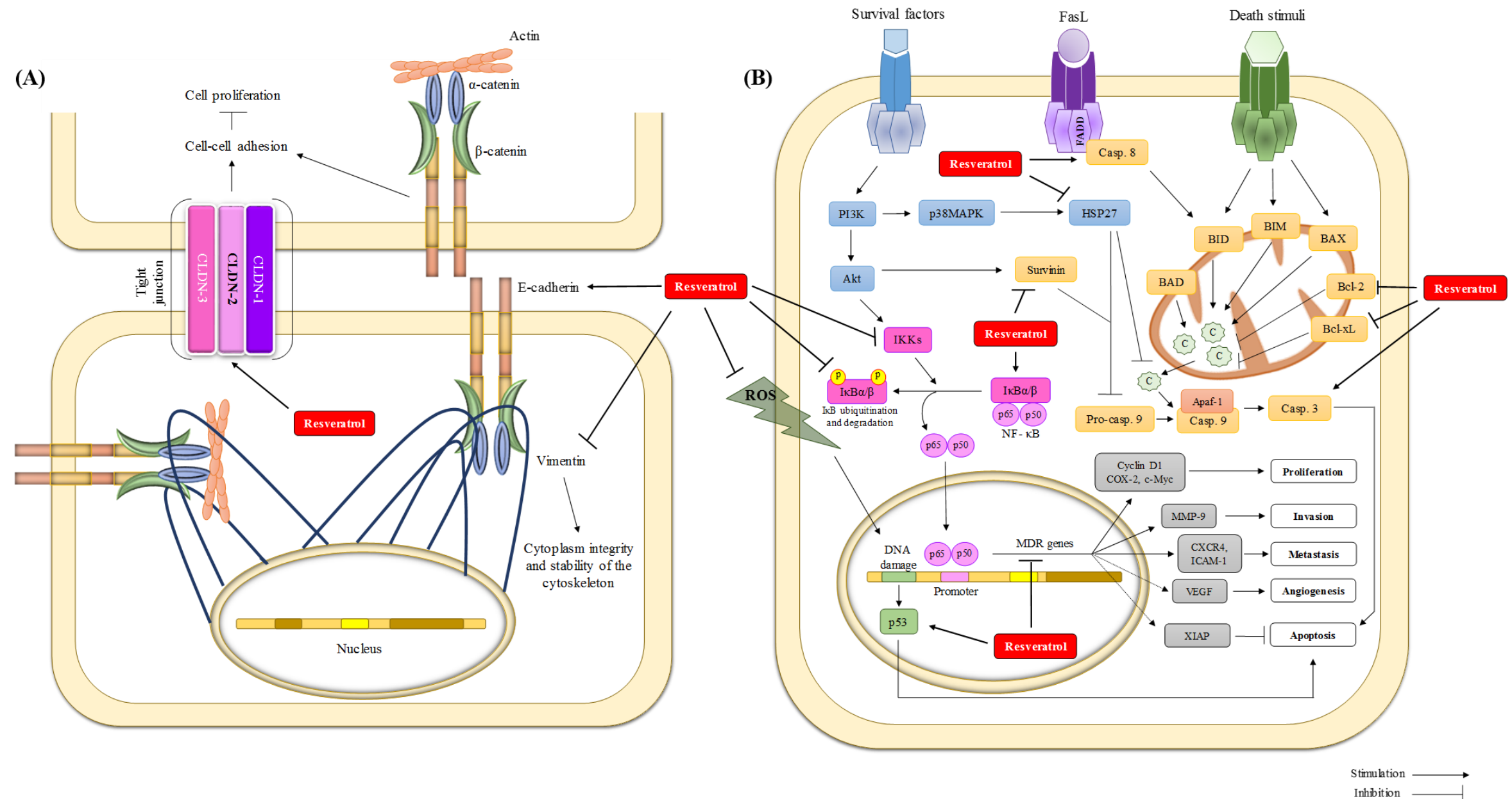

Figure 7. Molecular mechanisms of resveratrol-mediated chemosensitization. Resveratrol interferes in the expression of adhesion molecules and in the stability of the cell cycle (A), and modulates signaling pathways involved in apoptosis, cell proliferation, invasion, metastasis and angiogenesis (B). 


\subsection{Epigallocatechin-3-gallate}

Epigallocatechin-3-gallate (EGCG) is a major flavonoid found in green tea (Camelia sinensis) that possesses a broad spectrum of pharmacological activities, including antiangiogenic [153], anticarcinogenic [154], antimetastatic [155,156] and chemopreventive effects [157]. These properties are attributed to its antioxidant potential, cell signaling modulation, apoptosis induction, cell cycle arrest and inhibition of different MMPs (matrix metalloproteinases). In recent years, ECGC has been shown to be effective in sensitizing tumor cells to conventional chemotherapy. In fact, EGCG potentiates the antitumor effect of TRAIL (TNF $\alpha$-related apoptosis-inducing ligand) [47], 4-MU (4-methylumbelliferone) [59], taxane [51], IL-1Ra (IL-1 receptor antagonist) [52], capecitabine [53], vorinostat [54], cisplatin [48], tamoxifen [45,48], docetaxel [58] and doxorubicin [43] in various types of cancer, mainly breast $[45,59]$ and prostate cancer $[47,51,58]$.

In vitro and in vivo assays have demonstrated that EGCG enhances the antitumor effect of other drugs by inducing apoptosis. In general, EGCG up-regulates apoptotic proteins (e.g. BAD, BAK, BAX, caspases 3, 6, 7, 8 and 9) and down-regulates antiapoptotic factors (e.g. Bcl-2, Bcl-xL, XIAP, CIAP-1, survinin and Smac/Diablo) [47, 54, 58]. EGCG also induces the expression of genes that are directly associated with cell cycle arrest and apoptosis, such as p53, p73 and p21 [51].

Several studies have demonstrated that EGCG synergistically inhibits biomarkers associated with angiogenesis (e.g. VEGF, angiopoietin 1 and 2), invasion and metastasis (MMP-2, 3, and 9) [47, 52-54], improving the performance of chemotherapy in reducing tumor weight and/or volume in xenograft models [53]. Although inhibition of the NF- $\mathrm{B}$ pathway does not appear to be directly involved in the mechanism of EGCG-induced tumor cell sensitization, this flavonoid inhibits the Akt pathway, indirectly resulting in lower expression of factors associated with cell proliferation, invasion, metastasis, angiogenesis, and apoptosis. In addition, Wang et al. [58] showed that EGCG combined with quercetin inhibits STAT3 (signal transducer and activator of transcription 3) expression, contributing to sensitization of prostate cancer cells to docetaxel. In the same study, the authors also demonstrated the potential of these flavonoids to block MRP1 (multidrug resistance-associated protein 1), increasing the residence time of docetaxel in tumor cells. All mechanisms involved in the sensitization of tumor cells by EGCG are summarized in figure 8. 


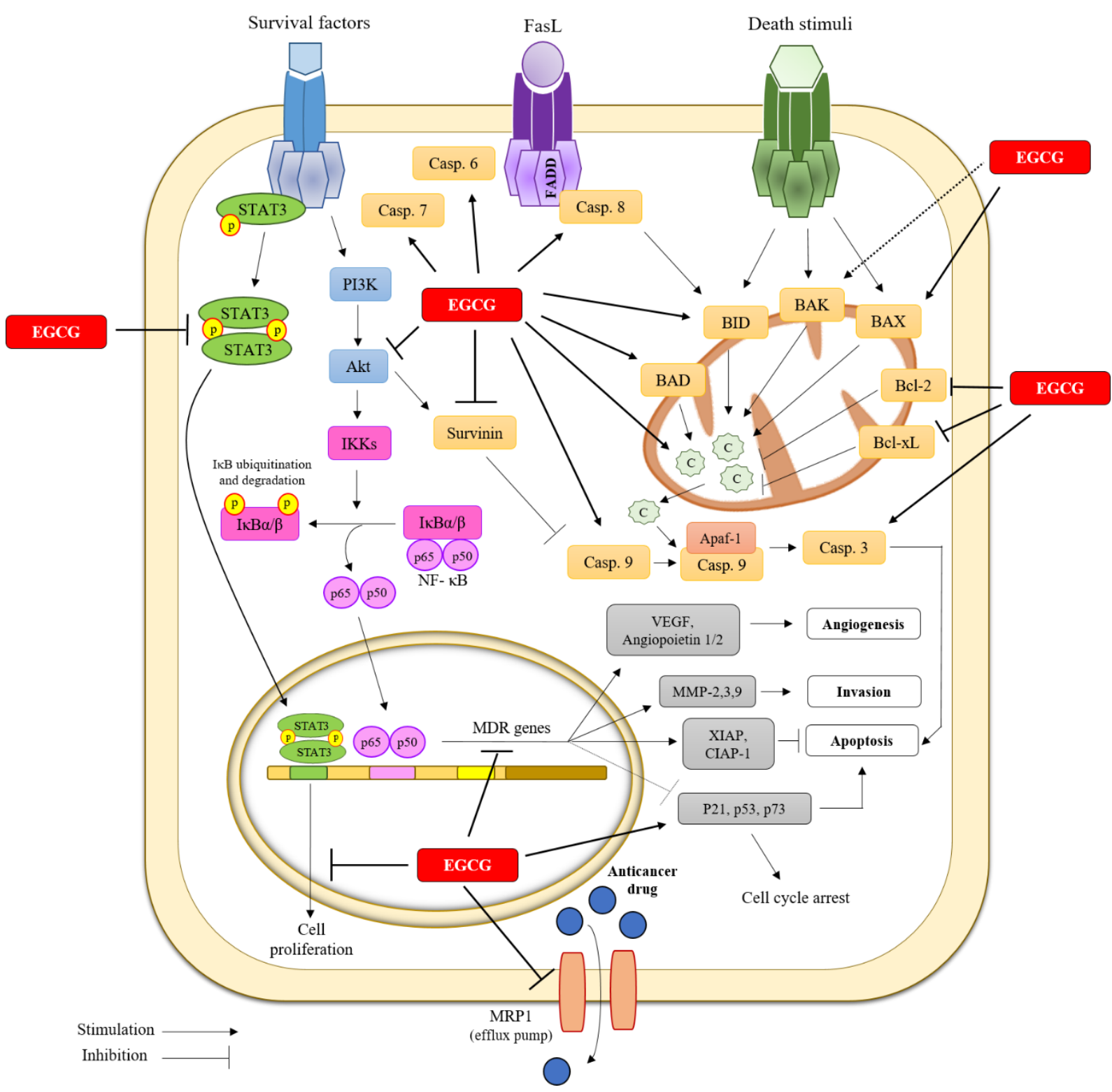

Figure 8. Molecular mechanisms of epigallocatechin-3-gallate (EGCG)-mediated chemosensitization. EGCG modulates signaling pathways involved in apoptosis, cell proliferation, invasion, metastasis and angiogenesis

\section{Conclusion}

This systematic review unified information from the literature on the use of natural compounds as chemosensitizers in cancer therapy. In vitro and in vivo studies demonstrated that natural products act synergistically with drugs traditionally used in cancer therapy, enhancing their antitumor efficacy through various mechanisms, including induction of apoptosis and inhibition of cell proliferation, invasion, metastasis, and angiogenesis. Although the in vivo tests presented moderate 
methodological quality, this report highlights the potential of natural products as anticancer drug candidates in future clinical research for combinatorial treatments. Considering that chemosensitization of cancer cells by natural products is a recent strategy and that only few resources have been explored at the moment, this research field should be expanding rapidly in the coming years and provide efficient alternatives to manage tumor chemoresistance.

\section{ACKNOWLEDGEMENTS}

We are grateful to the French Ministry of Research and Higher Education for funding RGOJ's PhD grant. We thank the French cancer league (Comite 17 de la Ligue Nationale contre le Cancer) for financial support and the "Cancéropôle Grand Ouest, axe Valorisation des produits de la mer en cancérologie" and Brazilian Society of Pharmacognosy for scientific support.

\section{CONFLICT OF INTEREST}

The authors declare that they have no conflict of interest.

\section{REFERENCES}

[1] J.S. You, P.A. Jones, Cancer Genetics and Epigenetics: Two Sides of the Same Coin? Cancer Cell. 22 (2012) 9-20. doi: 10.1016/j.ccr.2012.06.008.

[2] T.J. Harris, F. McCormick, The molecular pathology of cancer, Nat. Rev. Clin. Oncol. 7 (2010) 251-265. doi: 10.1038/nrclinonc.2010.41.

[3] D. Hanahan, R.A. Weinberg, Hallmarks of cancer: the next generation, Cell. 144 (2011) 646-674. doi: 10.1016/j.cell.2011.02.013.

[4] J.W. Shay, W.E. Wright, Role of telomeres and telomerase in cancer, Semin. Cancer. Biol. 21 (2011) 349-353. doi: 10.1016/j.semcancer.2011.10.001.

[5] M. Hassan, H. Watari, A. AbuAlmaaty, et al., Apoptosis and Molecular Targeting Therapy in Cancer, Biomed. Res. Int. 2014 (2014) 150845. doi: $10.1155 / 2014 / 150845$.

[6] Y.A. Fouad, C. Aanei, Revisiting the hallmarks of cancer, Am. J. Cancer Res. 7 (2017) 1016-1036.

[7] R.S.Y. Wong, Apoptosis in cancer: from pathogenesis to treatment, J. Exp. Clin. Cancer Res. 30 (2011) 87. doi: 10.1186/1756-9966-30-87. 
[8] L. Zitvogel, L. Galluzzi, M.J. Smyth, et al., Mechanism of action of conventional and targeted anticancer therapies: reinstating immunosurveillance, Immunity. 39 (2013) 74-88. doi: 10.1016/j.immuni.2013.06.014.

[9] T. Otto, P. Sicinski, Cell cycle proteins as promising targets in cancer therapy, Nat. Rev. Cancer. 17 (2017) 93-115. doi: 10.1038/nrc.2016.138.

[10] L. Galluzzi, O. Kepp, M.G.V. Heiden, et al., Metabolic targets for cancer therapy, Nat. Rev. Drug Discov. 12 (2013) 829-846. doi: 10.1038/nrd4145.

[11] G. Housman, S. Byler, S. Heerboth, et al., Drug resistance in cancer: an overview, Cancers (Basel). 6 (2014) 1769-1792. doi: 10.3390/cancers6031769.

[12] I.A. Cree, P. Charlton, Molecular chess? Hallmarks of anti-cancer drug resistance, BMC Cancer. 17 (2017) 10. doi: 10.1186/s12885-016-2999-1

[13] C. Holohan, S. Van Schaeybroeck, D.B. Longley, et al., Cancer drug resistance: an evolving paradigm, Nat. Rev. Cancer. 13 (2013) 714-726. doi: 10.1038/nrc3599.

[14] S. Shukla, A. Mehta, Anticancer potential of medicinal plants and their phytochemicals: a review, Braz. J. Bot. 38 (2015) 199-210. doi: $10.1007 / \mathrm{s} 40415-015-0135-0$

[15] V. Ruiz-Torres, J.A. Encinar, M. Herranz-López, et al., An Updated Review on Marine Anticancer Compounds: The Use of Virtual Screening for the Discovery of Small-Molecule Cancer Drugs, Molecules. 22 (2017) E1037. doi: 10.3390/molecules22071037.

[16] S. Sukhdev, B. Sharma, S.S. Kanwar, et al., Lead Phytochemicals for Anticancer Drug Development, Front. Plant. Sci. 7 (2016) 1667. doi: 10.3389/fpls.2016.01667.

[17] C. Calcabrini, E. Catanzaro, A. Bishayee, et al., Marine Sponge Natural Products with Anticancer Potential: An Updated Review, Mar. Drugs. 15 (2017) E310. doi: $10.3390 / \mathrm{md} 15100310$.

[18] R. Kotecha, A. Takami, J.L. Espinoza, Dietary phytochemicals and cancer chemoprevention: a review of the clinical evidence, Oncotarget. 7 (2016) 52517-52529. doi: 10.18632/oncotarget.9593.

[19] H. Wang, T.O. Khor, L. Shu, et al., Plants Against Cancer: A Review on Natural Phytochemicals in Preventing and Treating Cancers and Their Druggability, Anticancer Agents Med. Chem. 12 (2012) 1281-1305. 
[20] B.S. Vinod, T.T. Maliekal, R.J. Anto, Phytochemicals as chemosensitizers: from molecular mechanism to clinical significance, Antioxid. Redox. Signal. 18 (2013) 1307-1348. doi: 10.1089/ars.2012.4573.

[21] P. Dandawate, S. Padhye, A. Ahmad, et al., Novel strategies targeting cancer stem cells through phytochemicals and their analogs, Drug. Deliv. Transl. Res. 3 (2013) 165-182. doi: 10.1007/s13346-012-0079-x.

[22] S.A. Hussain, A.A. Sulaiman, C. Balch, et al., Natural Polyphenols in Cancer Chemoresistance, Nutr. Cancer. 6 (2016) 879-891. doi: 10.1080/01635581.2016.1192201.

[23] G. Jacquemin, S. Shirley, O. Micheau, Combining naturally occurring polyphenols with TNF-related apoptosis-inducing ligand: a promising approach to kill resistant cancer cells? Cell. Mol. Life. Sci. 67 (2010) 3115-3130. doi: 10.1007/s00018-010-0407-6.

[24] S.C Gupta, R. Kannappan, S. Reuter, et al., Chemosensitization of tumors by resveratrol, Ann. N. Y. Acad. Sci. 1215 (2011) 150-160. doi: 10.1111/j.17496632.2010.05852.x.

[25] D. Moher, A. Liberati, J. Tetzlaff, et al., Preferred reporting items for systematic reviews and meta-analyses: the PRISMA statement, PLoS Med. 6 (2009) e1000097. doi: 10.1371/journal.pmed.1000097.

[26] C.R. Hooijmans, M.M. Rovers, R.B. de Vries, et al., SYRCLE's risk of bias tool for animal studies, BMC Med. Res. Methodol. 14 (2014) 43. doi: 10.1186/14712288-14-43.

[27] P.S. Siqueira-Lima, J.C. Silva, J.S.S. Quintans, et al., Natural products assessed in animal models for orofacial pain - a systematic review, Braz. J. Pharmacog. 27 (2017) 124-134. doi: 10.1016/j.bjp.2016.06.005.

[28] M.Q. Song, J.S. Zhu, J.L. Chen, et al., Synergistic effect of oxymatrine and angiogenesis inhibitor NM-3 on modulating apoptosis in human gastric cancer cells, World J. Gastroenterol. 13 (2007) 1788-1793. doi: 10.3748/wjg.v13.i12.1788

[29] S. Banerjee, Z. Wang, D. Kong, et al., 3,3'-Diindolylmethane enhances chemosensitivity of multiple chemotherapeutic agents in pancreatic cancer, Cancer Res. 69 (2009) 5592-5600. doi: 10.1158/0008-5472.CAN-09-0838.

[30] B. Sung, K.S. Ahn, B.B. Aggarwal, Noscapine, a benzylisoquinoline alkaloid, sensitizes leukemic cells to chemotherapeutic agents and cytokines by 
modulating the NF-kappaB signaling pathway, Cancer Res. 70 (2010) 32593268. doi: 10.1158/0008-5472.CAN-09-4230.

[31] M.B. Chougule, A. Patel, P. Sachdeva, et al., Enhanced anticancer activity of gemcitabine in combination with noscapine via antiangiogenic and apoptotic pathway against non-small cell lung cancer, PLoS One, 6 (2011) e27394. doi: 10.1371/journal.pone.0027394.

[32] N. Tong, J. Zhang, Y. Chen, et al., Berberine sensitizes mutliple human cancer cells to the anticancer effects of doxorubicin in vitro, Oncol. Lett. 3 (2012) 1263-1267. doi: 10.3892/ol.2012.644.

[33] Q. Qi, X. Liu, S. Li, et al., Synergistic suppression of noscapine and conventional chemotherapeutics on human glioblastoma cell growth, Acta. Pharmacol. Sin. 34 (2013) 930-938. doi: 10.1038/aps.2013.40.

[34] J. Wang, Z.R. Yang, W.G. Dong, et al., Cooperative inhibitory effect of sinomenine combined with 5-fluorouracil on esophageal carcinoma, World J. Gastroenterol. 19 (2013) 8292-8300. doi: 10.3748/wjg.v19.i45.8292.

[35] N. Guo, A. Yan, X. Gao, et al., Berberine sensitizes rapamycin- mediated human hepatoma cell death in vitro, Mol. Med. Rep. 10 (2014) 3132-3138. doi: 10.3892/mmr.2014.2608.

[36] Y. Liu, T. Bi, W. Dai, et al., Oxymatrine synergistically enhances the inhibitory effect of 5-fluorouracil on hepatocellular carcinoma in vitro and in vivo, Tumour Biol. 37 (2016) 7589-7597. doi: 10.1007/s13277-015-4642-1.

[37] R. Doddapaneni, K. Patel, N. Chowdhury, et al., Noscapine chemosensitization enhances docetaxel anticancer activity and nanocarrier uptake in triple negative breast cancer, Exp. Cell Res. 346 (2016) 65-73. doi: 10.1016/j.yexcr.2016.05.006.

[38] Y. Zhao, Z. Jing, Y. Li, et al., Berberine in combination with cisplatin suppresses breast cancer cell growth through induction of DNA breaks and caspase-3-dependent apoptosis, Oncol. Rep. 36 (2016) 567-572. doi: 10.3892/or.2016.4785.

[39] P. Rajendran, F. Li, K.A. Manu, et al., $\gamma$-Tocotrienol is a novel inhibitor of constitutive and inducible STAT3 signalling pathway in human hepatocellular carcinoma: potential role as an antiproliferative, pro-apoptotic and chemosensitizing agent, Br. J. Pharmacol. 163 (2011) 283-298. doi: 10.1111/j.1476-5381.2010.01187.x. 
[40] Y.Z. Liu, C.M. Yang, J.Y. Chen, et al., Alpha-carotene inhibits metastasis in Lewis lung carcinoma in vitro, and suppresses lung metastasis and tumor growth in combination with taxol in tumor xenografted C57BL/6 mice, J. Nutr. Biochem. 26 (2015) 607-615. doi: 10.1016/j.jnutbio.2014.12.012.

[41] Y. Zhang, X. Zhu, T. Huang, et al., $\beta$-Carotene synergistically enhances the antitumor effect of 5-fluorouracil on esophageal squamous cell carcinoma in vivo and in vitro, Toxicol. Lett. 261 (2016) 49-58. doi: 10.1016/j.toxlet.2016.08.010.

[42] S.M. Kim, J.H. Lee, G. Sethi, et al., Bergamottin, a natural furanocoumarin obtained from grapefruit juice induces chemosensitization and apoptosis through the inhibition of STAT3 signaling pathway in tumor cells, Cancer Lett. 354 (2014) 153-163. doi: 10.1016/j.canlet.2014.08.002.

[43] G. Stammler, M. Volm, Green tea catechins (EGCG and EGC) have modulating effects on the activity of doxorubicin in drug-resistant cell lines, Anticancer Drugs. 8 (1997) 265-268.

[44] S. Dhanalakshmi, P. Agarwal, L.M. Glode, et al., Silibinin sensitizes human prostate carcinoma DU145 cells to cisplatin- and carboplatin-induced growth inhibition and apoptotic death, Int. J. Cancer. 106 (2003) 699-705.

[45] K. Chisholm, B.J. Bray, R.J. Rosengren, Tamoxifen and epigallocatechin gallate are synergistically cytotoxic to MDA-MB-231 human breast cancer cells, Anticancer Drugs. 15 (2004) 889-897.

[46] X.H. Peng, P. Karna, R.M. O'Regan, Down-regulation of inhibitor of apoptosis proteins by deguelin selectively induces apoptosis in breast cancer cells, Mol. Pharmacol. 71 (2007) 101-111.

[47] I.A. Siddiqui, A. Malik, V.M. Adhami, et al., Green tea polyphenol EGCG sensitizes human prostate carcinoma LNCaP cells to TRAIL-mediated apoptosis and synergistically inhibits biomarkers associated with angiogenesis and metastasis, Oncogene. 27 (2008) 2055-2063.

[48] A. Shervington, V. Pawar, S. Menon, et al., The sensitization of glioma cells to cisplatin and tamoxifen by the use of catechin, Mol. Biol. Rep. 36 (2009) 11811186. doi: 10.1007/s11033-008-9295-3.

[49] F.Y. Zhang, G.J. Du, L. Zhang, et al., Naringenin enhances the anti-tumor effect of doxorubicin through selectively inhibiting the activity of multidrug resistanceassociated proteins but not P-glycoprotein, Pharm. Res. 26 (2009) 914-925. doi: 10.1007/s11095-008-9793-y. 
[50] C.Y. Jin, C. Park, H.J. Hwang, et al., Naringenin up-regulates the expression of death receptor 5 and enhances TRAIL-induced apoptosis in human lung cancer A549 cells, Mol. Nutr. Food Res. 55 (2011) 300-309. doi: 10.1002/mnfr.201000024.

[51] M.E. Stearns, M. Wang, Synergistic Effects of the Green Tea Extract Epigallocatechin-3-gallate and Taxane in Eradication of Malignant Human Prostate Tumors, Transl. Oncol. 4 (2011) 147-156.

[52] A.S. Hönicke, S.A. Ender, J. Radons, Combined administration of EGCG and IL-1 receptor antagonist efficiently downregulates IL-1-induced tumorigenic factors in U-2 OS human osteosarcoma cells, Int. J. Oncol. 41 (2012) 753-758. doi: 10.3892/ijo.2012.1498.

[53] H. Wu, Y. Xin, C. Xu, et al., Capecitabine combined with (-)-epigallocatechin-3gallate inhibits angiogenesis and tumor growth in nude mice with gastric cancer xenografts, Exp. Ther. Med. 3 (2012) 650-654. doi: 10.3892/etm.2012.448.

[54] T.W. Kwak, D.H. Kim, C.W. Chung, et al., Synergistic Anticancer Effects of Vorinostat and Epigallocatechin-3-Gallate against HuCC-T1 Human Cholangiocarcinoma Cells, Evid. Based Complementary Altern. Med. 2013 (2013) 18515. doi: 10.1155/2013/185158.

[55] R. Suzuki, Y. Kang, X. Li, et al., Genistein potentiates the antitumor effect of 5Fluorouracil by inducing apoptosis and autophagy in human pancreatic cancer cells, Anticancer Res. 34 (2014) 4685-4692.

[56] L. Wang, J. Feng, X. Chen, et al., Myricetin enhance chemosensitivity of 5fluorouracil on esophageal carcinoma in vitro and in vivo, Cancer Cell Int. 14 (2014) 71. doi: 10.1186/s12935-014-0071-2.

[57] M.S. Abaza, K.Y. Orabi, E. Al-Quattan, et al., Growth inhibitory and chemosensitization effects of naringenin, a natural flavanone purified from Thymus vulgaris, on human breast and colorectal cancer, Cancer Cell Int. 15 (2015) 46. doi: 10.1186/s12935-015-0194-0.

[58] P. Wang, S.M. Henning, D. Heber, et al., Sensitization to docetaxel in prostate cancer cells by green tea and quercetin, J. Nutr. Biochem. 26 (2015) 408-415. doi: 10.1016/j.jnutbio.2014.11.017.

[59] J.A. García-Vilas, A.R. Quesada, M.A. Medina, Screening of synergistic interactions of epigallocatechin-3-gallate with antiangiogenic and antitumor compounds, Synergy. 3 (2016) 5-13. doi: 10.1016/j.synres.2016.05.001. 
[60] T. Krajnović, G.N. Kaluđerović, L.A. Wessjohann, et al., Versatile antitumor potential of isoxanthohumol: Enhancement of paclitaxel activity in vivo, Pharmacol. Res. 105 (2016) 62-73. doi: 10.1016/j.phrs.2016.01.011.

[61] S. Lin, K. Lei, W. Du, et al., Enhancement of oxaliplatin sensitivity in human colorectal cancer by hypericin mediated photodynamic therapy via ROS-related mechanism, Int. J. Biochem. Cell Biol. 71 (2016) 24-34. doi: 10.1016/j.biocel.2015.12.003.

[62] S. Lin, L. Yang, H. Shi, et al., Endoplasmic reticulum-targeting photosensitizer Hypericin confers chemo-sensitization towards oxaliplatin through inducing prodeath autophagy, Int. J. Biochem. Cell Biol. 87 (2017) 54-68. doi: 10.1016/j.biocel.2017.04.001.

[63] S. Anuchapreeda, P. Leechanachai, M.M. Smith, et al., Modulation of Pglycoprotein expression and function by curcumin in multidrug-resistant human KB cells, Biochem. Pharmacol. 64 (2002) 573-582.

[64] T.C. Hour, J. Chen, C.Y. Huang, et al., Curcumin enhances cytotoxicity of chemotherapeutic agents in prostate cancer cells by inducing p21(WAF1/CIP1) and C/EBPbeta expressions and suppressing NF-kappaB activation, Prostate. 51 (2002) 211-218.

[65] S. Fulda, K.M. Debatin, Sensitization for anticancer drug-induced apoptosis by the chemopreventive agent resveratrol, Oncogene. 23 (2004) 6702-6711.

[66] S.L. Wu, Z. J. Sun, L. Yu, et al., Effect of resveratrol and in combination with 5FU on murine liver cancer, World J. Gastroenterol. 10 (2004) 3048-3052.

[67] B.B. Aggarwal, S. Shishodia, Y. Takada, et al., Curcumin suppresses the paclitaxel-induced nuclear factor-kappaB pathway in breast cancer cells and inhibits lung metastasis of human breast cancer in nude mice, Clin. Cancer Res. 11 (2005) 7490-7498.

[68] S.V. Bava, V.T. Puliappadamba, A. Deepti, et al., Sensitization of taxol-induced apoptosis by curcumin involves down-regulation of nuclear factor-??B and the serine/threonine kinase Akt and is independent of tubulin polymerization, J. Biol. Chem. 280 (2005) 6301-6308. doi:10.1074/jbc.M410647200.

[69] L. Li, B. Ahmed, K. Mehta, R. Kurzrock, Liposomal curcumin with and without oxaliplatin: effects on cell growth, apoptosis, and angiogenesis in colorectal cancer, Mol. Cancer Ther. 6 (2007) 1276-1282. doi:10.1158/1535-7163.MCT06-0556. 
[70] K.S. Chen, Y.C. Hsiao, D.Y. Kuo, et al., Tannic acid-induced apoptosis and enhanced sensitivity to arsenic trioxide in human leukemia HL-60 cells, Leuk. Res. 33 (2009) 297-307. doi:10.1016/j.leukres.2008.08.006.

[71] K.B. Harikumar, A.B. Kunnumakkara, G. Sethi, et al., Resveratrol, a multitargeted agent, can enhance antitumor activity of gemcitabine in vitro and in orthotopic mouse model of human pancreatic cancer, Int. J. Cancer. 127 (2010) 257-268. doi:10.1002/ijc.25041.

[72] A.B. Kunnumakkara, P. Diagaradjane, P. Anand, et al., Curcumin sensitizes human colorectal cancer to capecitabine by modulation of cyclin D1, COX-2, MMP-9, VEGF and CXCR4 expression in an orthotopic mouse model, Int. J. Cancer. 125 (2009) 2187-2197. doi:10.1002/ijc.24593.

[73] Y. Yu, S.S. Kanwar, B.B. Patel, et al., Elimination of Colon Cancer Stem-Like Cells by the Combination of Curcumin and FOLFOX, Transl. Oncol. 2 (2009) 321-328. doi:10.1593/tlo.09193.

[74] W. Hartojo, A.L. Silvers, D.G. Thomas, et al., Curcumin promotes apoptosis, increases chemosensitivity, and inhibits nuclear factor kappaB in esophageal adenocarcinoma., Transl. Oncol. 3 (2010) 99-108. doi:10.1593/tlo.09235.

[75] S. V. Bava, C.N. Sreekanth, A.K.T. Thulasidasan, et al., Akt is upstream and MAPKs are downstream of NF-??B in paclitaxel-induced survival signaling events, which are down-regulated by curcumin contributing to their synergism, Int. J. Biochem. Cell Biol. 43 (2011) 331-341. doi:10.1016/j.biocel.2010.09.011.

[76] C.N. Sreekanth, S. V Bava, E. Sreekumar, et al., Molecular evidences for the chemosensitizing efficacy of liposomal curcumin in paclitaxel chemotherapy in mouse models of cervical cancer, Oncogene. 30 (2011) 3139-3152. doi:10.1038/onc.2011.23.

[77] A.M.M. Osman, H.M. Bayoumi, S.E. Al-Harthi, et al., Modulation of doxorubicin cytotoxicity by resveratrol in a human breast cancer cell line, Cancer Cell Int. 12 (2012) 47. doi:10.1186/1475-2867-12-47.

[78] E.M. Saleh, R.A. El-awady, N.A. Eissa, et al., Antagonism between curcumin and the topoisomerase II inhibitor etoposide: A study of DNA damage, cell cycle regulation and death pathways, Cancer Biol. Ther. 13 (2012) 1058-1071. doi:10.4161/cbt.21078.

[79] H. Wang, Q.R. Geng, L. Wang, et al., Curcumin potentiates antitumor activity of L-asparaginase via inhibition of the AKT signaling pathway in acute 
lymphoblastic leukemia., Leuk. Lymphoma. 53 (2012) 1376-82. doi:10.3109/10428194.2011.649478.

[80] F. Amiri, A.H. Zarnani, H. Zand, et al., Synergistic anti-proliferative effect of resveratrol and etoposide on human hepatocellular and colon cancer cell lines, Eur. J. Pharmacol. 718 (2013) 34-40. doi:10.1016/j.ejphar.2013.09.020.

[81] J. Díaz-Chávez, M.A. Fonseca-Sánchez, E. Arechaga-Ocampo, et al., Proteomic Profiling Reveals That Resveratrol Inhibits HSP27 Expression and Sensitizes Breast Cancer Cells to Doxorubicin Therapy, PLoS One. 8 (2013) 1-11. doi:10.1371/journal.pone.0064378.

[82] M. Shakibaei, A. Mobasheri, C. Lueders, et al., Curcumin Enhances the Effect of Chemotherapy against Colorectal Cancer Cells by Inhibition of NF- $\kappa \mathrm{B}$ and Src Protein Kinase Signaling Pathways, PLoS One. 8 (2013) 1-13. doi:10.1371/journal.pone.0057218.

[83] L.J. Carlson, B. Cote, A.W. Alani, et al., Polymeric micellar co-delivery of resveratrol and curcumin to mitigate in vitro doxorubicin-induced cardiotoxicity, J. Pharm. Sci. 103 (2014) 2315-2322. doi:10.1002/jps.24042.

[84] H. Qian, Y. Yang, X. Wang, Curcumin enhanced adriamycin-induced human liver-derived Hepatoma G2 cell death through activation of mitochondriamediated apoptosis and autophagy, Eur. J. Pharm. Sci. 43 (2011) 125-131. doi:10.1016/j.ejps.2011.04.002.

[85] C. Buhrmann, P. Shayan, P. Kraehe, et al., Resveratrol induces chemosensitization to 5-fluorouracil through up-regulation of intercellular junctions, Epithelial-to-mesenchymal transition and apoptosis in colorectal cancer, Biochem. Pharmacol. 98 (2015) 51-58. doi:10.1016/j.bcp.2015.08.105.

[86] B. Cote, L.J. Carlson, D.A. Rao, et al., Combinatorial resveratrol and quercetin polymeric micelles mitigate doxorubicin induced cardiotoxicity in vitro and in vivo, J. Control. Release. $213 \quad$ (2015) 128-133. doi:10.1016/j.jconrel.2015.06.040.

[87] M. Shakibaei, P. Kraehe, B. Popper, et al., Curcumin potentiates antitumor activity of 5-fluorouracil in a 3D alginate tumor microenvironment of colorectal cancer, BMC Cancer. 15 (2015) 250. doi:10.1186/s12885-015-1291-0.

[88] M.S.I. Abaza, M. Afzal, R.J. Al-Attiyah, et al., Methylferulate from Tamarix aucheriana inhibits growth and enhances chemosensitivity of human colorectal 
cancer cells: possible mechanism of action., BMC Complement. Altern. Med. 16 (2016) 384. doi:10.1186/s12906-016-1358-8.

[89] E. Ooko, T. Alsalim, B. Saeed, et al., Modulation of P-glycoprotein activity by novel synthetic curcumin derivatives in sensitive and multidrug-resistant T-cell acute lymphoblastic leukemia cell lines, Toxicol. Appl. Pharmacol. 305 (2016) 216-233. doi:10.1016/j.taap.2016.06.002.

[90] A.K. Tyagi, S. Prasad, M. Majeed, et al., Calebin A, a novel component of turmeric, suppresses NF- $\kappa \mathrm{B}$ regulated cell survival and inflammatory gene products leading to inhibition of cell growth and chemosensitization, Phytomedicine. 34 (2017) 171-181. doi:10.1016/j.phymed.2017.08.021.

[91] S.H. Jafri, J. Glass, R. Shi, et al., Thymoquinone and cisplatin as a therapeutic combination in lung cancer: In vitro and in vivo., J. Exp. Clin. Cancer Res. 29 (2010) 87. doi:10.1186/1756-9966-29-87.

[92] F. Li, P. Rajendran, G. Sethi, Thymoquinone inhibits proliferation, induces apoptosis and chemosensitizes human multiple myeloma cells through suppression of signal transducer and activator of transcription 3 activation pathway, Br. J. Pharmacol. 161 (2010) 541-554. doi:10.1111/j.14765381.2010.00874.x.

[93] S.K. Sandur, M.K. Pandey, B. Sung, et al., 5-hydroxy-2-methyl-1,4naphthoquinone, a vitamin K3 analogue, suppresses STAT3 activation pathway through induction of protein tyrosine phosphatase, SHP-1: potential role in chemosensitization., Mol. Cancer Res. 8 (2010) 107-18. doi:10.1158/15417786.MCR-09-0257.

[94] K. Effenberger-Neidnicht, R. Schobert, Combinatorial effects of thymoquinone on the anti-cancer activity of doxorubicin, Cancer Chemother. Pharmacol. 67 (2011) 867-874. doi: 10.1007/s00280-010-1386-X.

[95] Y. Wang, Y. Zhou, G. Jia, et al., Shikonin suppresses tumor growth and synergizes with gemcitabine in a pancreatic cancer xenograft model:

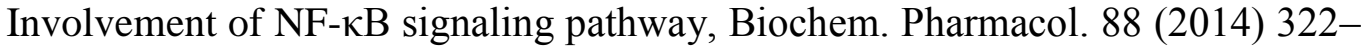
333. doi:10.1016/j.bcp.2014.01.041.

[96] W. Daqian, W. Chuandong, Q. Xinhua, et al., Chimaphilin inhibits proliferation and induces apoptosis in multidrug resistant osteosarcoma cell lines through insulin-like growth factor-I receptor (IGF-IR) signaling, Chem. Biol. Interact. 237 (2015) 25-30. doi:10.1016/j.cbi.2015.05.008. 
[97] G. He, G. He, R. Zhou, et al., Enhancement of cisplatin-induced colon cancer cells apoptosis by shikonin, a natural inducer of ROS in vitro and in vivo, Biochem. Biophys. Res. Commun. 469 (2016) 1075-1082. doi:10.1016/j.bbrc.2015.12.100.

[98] J. Song, Z. Zhao, X. Fan, et al., Shikonin potentiates the effect of arsenic trioxide against human hepatocellular carcinoma in vitro and in vivo, Oncotarget. 7 (2016). doi:10.18632/oncotarget.12041.

[99] Y. Wang, H.L. Lu, Y.D. Liu, et al., Cryptotanshinone sensitizes antitumor effect of paclitaxel on tongue squamous cell carcinoma growth by inhibiting the JAK/STAT3 signaling pathway, Biomed. Pharmacother. 95 (2017) 1388-1396. doi:10.1016/j.biopha.2017.09.062.

[100] C.H. Choi, G. Kang, Y.D. Min, Reversal of P-glycoprotein-mediated multidrug resistance by protopanaxatriol ginsenosides from Korean red ginseng, Planta Med. 69 (2003) 235-240. doi:10.1055/s-2003-38483.

[101] S.M. Kim, S.Y. Lee, J.S. Cho, et al., Hong, Combination of ginsenoside Rg3 with docetaxel enhances the susceptibility of prostate cancer cells via inhibition of NF-кB, Eur. J. Pharmacol. 631 (2010) 1-9. doi:10.1016/j.ejphar.2009.12.018.

[102] Z.J. Ming, Y. Hu, Y.H. Qiu, et al., Synergistic effects of $\beta$-aescin and 5fluorouracil in human hepatocellular carcinoma SMMC-7721 cells, Phytomedicine. 17 (2010) 575-580. doi:10.1016/j.phymed.2009.12.009.

[103] Y.W. Wang, S.J. Wang, Y.N. Zhou, et al., Escin augments the efficacy of gemcitabine through down-regulation of nuclear factor- $\kappa \mathrm{B}$ and nuclear factor$\kappa \mathrm{B}-$ regulated gene products in pancreatic cancer both in vitro and in vivo, J. Cancer Res. Clin. Oncol. 138 (2012) 785-797. doi:10.1007/s00432-012-1152-z.

[104] L.Q. Yang, B. Wang, H. Gan, et al., Enhanced oral bioavailability and antitumour effect of paclitaxel by 20(s)-ginsenoside $\operatorname{Rg} 3$ in vivo, Biopharm. Drug Dispos. 33 (2012) 425-436. doi: 10.1002/bdd.1806.

[105] H. Wang, Z. Zhai, N. Li, et al., Steroidal saponin of Trillium tschonoskii. Reverses multidrug resistance of hepatocellular carcinoma, Phytomedicine. 20 (2013) 985-991. doi:10.1016/j.phymed.2013.04.014.

[106] L. Chang, B. Huo, Y. Lv, et al., Ginsenoside Rg3 enhances the inhibitory effects of chemotherapy on esophageal squamous cell carcinoma in mice, Mol. Clin. Oncol. 2 (2014) 1043-1046. doi:10.3892/mco.2014.355. 
[107] Y.J. Lee, S. Lee, J.N. Ho, et al., Synergistic antitumor effect of ginsenoside Rg3 and cisplatin in cisplatin-resistant bladder tumor cell line, Oncol. Rep. 32 (2014) 1803-1808. doi:10.3892/or.2014.3452.

[108] Z. Liu, Q. Zheng, W. Chen, et al., Chemosensitizing effect of Paris Saponin I on Camptothecin and 10-hydroxycamptothecin in lung cancer cells via p38 MAPK, ERK, and Akt signaling pathways, Eur. J. Med. Chem. 125 (2017) 760-769. doi:10.1016/j.ejmech.2016.09.066.

[109] Z. Yuan, H. Jiang, X. Zhu, et al., Ginsenoside Rg3 promotes cytotoxicity of Paclitaxel through inhibiting $\mathrm{NF}-\kappa \mathrm{B}$ signaling and regulating $\mathrm{Bax} / \mathrm{Bcl}-2$ expression on triple-negative breast cancer, Biomed. Pharmacother. 89 (2017) 227-232. doi:10.1016/j.biopha.2017.02.038.

[110] T.J. Lee, H.J. Um, D.S. Min, et al., Withaferin A sensitizes TRAIL-induced apoptosis through reactive oxygen species-mediated up-regulation of death receptor 5 and down-regulation of c-FLIP, Free Radic. Biol. Med. 46 (2009) 1639-1649. doi:10.1016/j.freeradbiomed.2009.03.022.

[111] W. Chen, A. Leiter, D. Yin, et al., Cucurbitacin B inhibits growth, arrests the cell cycle, and potentiates antiproliferative efficacy of cisplatin in cutaneous squamous cell carcinoma cell lines, Int. J. Oncol. 37 (2010) 737-743.

[112] G.B. Iwanski, D.H. Lee, S. En-Gal, et al., Cucurbitacin B, a novel in vivo potentiator of gemcitabine with low toxicity in the treatment of pancreatic cancer, Br. J. Pharmacol. 160 (2010) 998-1007. doi:10.1111/j.14765381.2010.00741.x.

[113] D.H. Lee, N.H. Thoennissen, C. Goff, et al., Synergistic effect of low-dose cucurbitacin B and low-dose methotrexate for treatment of human osteosarcoma, Cancer Lett. 306 (2011) 161-170. doi:10.1016/j.canlet.2011.03.001.

[114] S.M. Cohen, R. Mukerji, B.N. Timmermann, et al., A novel combination of withaferin A and sorafenib shows synergistic efficacy against both papillary and anaplastic thyroid cancers, Am. J. Surg. 204 (2012) 895-901. doi:10.1016/j.amjsurg.2012.07.027.

[115] M.Y. Fong, S. Jin, M. Rane, et al., Withaferin a synergizes the therapeutic effect of doxorubicin through ROS-mediated autophagy in ovarian cancer, PLoS One. 7 (2012) 1-16. doi:10.1371/journal.pone.0042265. 
[116] F.F. El-Senduny, F.A. Badria, A.M. EL-Waseef, et al., Approach for chemosensitization of cisplatin-resistant ovarian cancer by cucurbitacin B, Tumor Biol. 37 (2016) 685-698. doi:10.1007/s13277-015-3773-8.

[117] X. Li, F. Zhu, J. Jiang, et al., Synergistic antitumor activity of withaferin A combined with oxaliplatin triggers reactive oxygen species-mediated inactivation of the PI3K/AKT pathway in human pancreatic cancer cells, Cancer Lett. 357 (2015) 219-230. doi:10.1016/j.canlet.2014.11.026.

[118] M. Ben-Eltriki, S. Deb, H. Adomat, et al., Calcitriol and 20(S)-protopanaxadiol synergistically inhibit growth and induce apoptosis in human prostate cancer cells, J. Steroid Biochem. Mol. Biol. 158 (2016) 207-219. doi:10.1016/j.jsbmb.2015.12.002.

[119] M.L. Holland, J.A. Panetta, J.M. Hoskins, et al., The effects of cannabinoids on P-glycoprotein transport and expression in multidrug resistant cells, Biochem. Pharmacol. 71 (2006) 1146-1154. doi:10.1016/j.bcp.2005.12.033.

[120] S. Sieber, G. Gdynia, W. Roth, et al., Combination treatment of malignant B cells using the anti-CD20 antibody rituximab and the anti-malarial artesunate, Int. J. Oncol. 35 (2009) 149-158.

[121] Y. He, F. Liu, L. Zhang, et al., Growth inhibition and apoptosis induced by lupeol, a dietary triterpene, in human hepatocellular carcinoma cells., Biol. Pharm. Bull. 34 (2011) 517-22. doi:10.1248/bpb.34.517.

[122] R. Kannaiyan, H.S. Hay, P. Rajendran, et al., Celastrol inhibits proliferation and induces chemosensitization through down-regulation of NF- $\kappa \mathrm{B}$ and STAT3 regulated gene products in multiple myeloma cells, Br. J. Pharmacol. 164 (2011) 1506-1521. doi:10.1111/j.1476-5381.2011.01449.x.

[123] S. Torres, M. Lorente, F. Rodriguez-Fornes, et al., A Combined Preclinical Therapy of Cannabinoids and Temozolomide against Glioma, Mol. Cancer Ther. 10 (2011) 90-103. doi:10.1158/1535-7163.MCT-10-0688.

[124] S. Prasad, V.R. Yadav, B. Sung, et al., Ursolic acid inhibits growth and metastasis of human, colorectal cancer in an orthotopic nude mouse model by targeting multiple cell signaling pathways: Chemosensitization with capecitabine, Clin. Cancer Res. 18 (2012) 4942-4953. doi:10.1158/10780432.CCR-11-2805. 
[125] J. Tong, S. Yin, Y. Dong, et al., Pseudolaric Acid B Induces Caspase-Dependent Apoptosis and Autophagic Cell Death in Prostate Cancer Cells, Phytother. Res. 27 (2013) 885-891. doi: 10.1002/ptr.4808.

[126] Y. Wang, Y. Zhou, H. Zhou, et al., Pristimerin Causes G1 Arrest, Induces Apoptosis, and Enhances the Chemosensitivity to Gemcitabine in Pancreatic Cancer Cells, PLoS One. 7 (2012) 1-12. doi:10.1371/journal.pone.0043826.

[127] E. Butturini, A.C. Prati, G. Chiavegato, et al., Mild oxidative stress induces Sglutathionylation of STAT3 and enhances chemosensitivity of tumoural cells to chemotherapeutic drugs, Free Radic. Biol. Med. 65 (2013) 1322-1330. doi:10.1016/j.freeradbiomed.2013.09.015.

[128] F. Liu, Y. He, Y. Liang, et al., PI3-kinase inhibition synergistically promoted the anti-tumor effect of lupeol in hepatocellular carcinoma., Cancer Cell Int. 13 (2013) 108. doi:10.1186/1475-2867-13-108.

[129] O.A. Bamodu, W.C. Huang, D.T.W. Tzeng, et al., Ovatodiolide sensitizes aggressive breast cancer cells to doxorubicin, eliminates their cancer stem celllike phenotype, and reduces doxorubicin-associated toxicity, Cancer Lett. 364 (2015) 125-134. doi:10.1016/j.canlet.2015.05.006.

[130] Y. Liu, T. Bi, W. Dai, et al., Lupeol enhances inhibitory effect of 5-fluorouracil on human gastric carcinoma cells, Naunyn. Schmiedebergs. Arch. Pharmacol. 389 (2016) 477-484. doi:10.1007/s00210-016-1221-y.

[131] M.A. Reis, O.B. Ahmed, G. Spengler, et al., Jatrophane diterpenes and cancer multidrug resistance - ABCB1 efflux modulation and selective cell death induction, $\quad$ Phytomedicine. $23 \quad$ (2016) 968-978. doi:10.1016/j.phymed.2016.05.007.

[132] B.C. Baguley, Multiple drug resistance mechanisms in cancer, Mol. Biotechnol. 46 (2010) 308-316. doi: 10.1007/s12033-010-9321-2.

[133] Q. Wu, Z. Yang, Y. Nie, et al., Multi-drug resistance in cancer chemotherapeutics: mechanisms and lab approaches, Cancer Lett. 347 (2014) 159-166. doi: 10.1016/j.canlet.2014.03.013.

[134] W.Y. Wu, J.J. Hou, H.L. Long, et al., TCM-based new drug discovery and development in China, Chin. J. Nat. Med. 12 (2014) 241-250. doi: 10.1016/S1875-5364(14)60050-9. 
[135] J. Liu, S. Wang, Y. Zhang, et al., Traditional Chinese medicine and cancer: History, present situation, and development, Thorac. Cancer. 6 (2015) 561-569. doi: $10.1111 / 1759-7714.12270$.

[136] A. Richmond, Y. Su, Mouse xenograft models vs GEM models for human cancer therapeutics, Dis. Model Mech. 1 (2008) 78-82. doi: 10.1242/dmm.000976.

[137] J. Jung, Human Tumor Xenograft Models for Preclinical Assessment of Anticancer Drug Development, Toxicol. Res. 30 (2014) 1-5. doi: 10.5487/TR.2014.30.1.001.

[138] M.F. Festing, D.G. Altman, Guidelines for the design and statistical analysis of experiments using laboratory animals, ILAR J. 43 (2002) 244-258.

[139] J.A. Hirst, J. Howick, J.K. Aronson, et al., The need for randomization in animal trials: an overview of systematic reviews, Plos One. 9 (2014) e98856. doi: 10.1371/journal.pone.0098856.

[140] P.J. Karanicolas, F. Farrokhyar, M. Bhandari, Blinding: Who, what, when, why, how? Can. J. Surg. 53 (2010) 345-348.

[141] J.E. Aguilar-Nascimento, Fundamental steps in experimental design for animal studies, Acta. Cir. Bras. 20 (2005) 2-8.

[142] V.S. Govindarajan, Turmeric: chemistry, technology, and quality, Crit Rev Food Sci Nutr. 12 (1980) 199-301.

[143] N. Chainani-Wu, Safety and anti-inflammatory activity of curcumin: a component of tumeric (Curcuma longa), J. Altern. Complement. Med. 9 (2003) $161-168$.

[144] B. Chandran, A. Goel, A randomized, pilot study to assess the efficacy and safety of curcumin in patients with active rheumatoid arthritis, Phytother. Res. 26 (2012) 1719-1725. doi: 10.1002/ptr.4639.

[145] S.C. Gupta, S. Patchva, B.B. Aggarwal, Therapeutic Roles of Curcumin: Lessons Learned from Clinical Trials, AAPS J. 15 (2013) 195-218. doi: 10.1208/s12248-012-9432-8.

[146] M. Karin, Nuclear factor- $\kappa B$ in cancer development and progression, Nature. 441 (2006) 431-436.

[147] I. Vivanco, C.L. Sawyers, The phosphatidylinositol 3-Kinase AKT pathway in human cancer, Nat. Rev. Cancer. 2 (2002) 489-501. 
[148] E.M. Varoni, A.F.L. Faro, J. Sharifi-Rad, et al., Anticancer Molecular Mechanisms of Resveratrol, Front. Nutr. 3 (2016). doi: 10.3389/fnut.2016.00008.

[149] N.C. Whitlock, S.J. Baek, The anticancer effects of resveratrol - Modulation of transcription factors, Nutr. Cancer. 64 (2012) 493-502. doi: 10.1080/01635581.2012.667862.

[150] J.K. Aluyen, Q.N. Ton, T. Tran, et al., Resveratrol: potential as anticancer agent, J. Diet Suppl. 9 (2012) 45-56. doi: 10.3109/19390211.2011.650842.

[151] J. Acunzo, C. Andrieu, V. Baylot, et al., Hsp27 as a therapeutic target in cancers, Curr. Drug Targets. 15 (2014) 423-431.

[152] A. Satelli, S. Li, Vimentin in cancer and its potential as a molecular target for cancer therapy, Cell. Mol. Life Sci. 68 (2011) 3033-3046. doi: 10.1007/s00018011-0735-1.

[153] Y. Sakamoto, N. Terashita, T. Muraguchi, et al., Effects of epigallocatechin-3gallate (EGCG) on A549 lung cancer tumor growth and angiogenesis, Biosci. Biotechnol. Biochem. 77 (2013) 1799-1803.

[154] H. Fujiki, S. Yoshizawa, T. Horiuchi, et al., Anticarcinogenic effects of (-)epigallocatechin gallate, Prev. Med. 21 (1992) 503-509.

[155] C. Braicu, C.D. Gherman, A. Irimie, et al., Epigallocatechin-3-Gallate (EGCG) inhibits cell proliferation and migratory behaviour of triple negative breast cancer cells, J. Nanosci. Nanotechnol. 13 (2013) 632-637.

[156] S. Shankar, S. Ganapathy, S.R. Hingorani, et al., EGCG inhibits growth, invasion, angiogenesis and metastasis of pancreatic cancer, Front. Biosci. 1 (2008) 440-452.

[157] J.D. Lambert, C.S. Yang, Mechanisms of cancer prevention by tea constituents, J. Nutr. 133 (2013) 3262S-3267S. 